\title{
Impacts of acquisition and reconstruction parameters on the absolute technetium quantification of the cadmium-zinc- telluride-based SPECT/CT system: a phantom study
}

Ruyi Zhang, Miao Wang, Yaqian Zhou, Shen Wang, Yiming Shen, Ning Li, Peng Wang, Jian Tan, Zhaowei Meng ${ }^{*+}$ (D) and Qiang Jia ${ }^{*+}$

\section{*Correspondence:}

zmeng@tmu.edu.cn;

jiaqiang4321@163.com

†Zhaowei Meng and Qiang

Jia contributed equally to

this study.

Department of Nuclear

Medicine, Tianjin Medical

University General

Hospital, Anshan Road

No. 154, Heping District,

Tianjin 300052, People's

Republic of China

\begin{abstract}
Background: The digital cadmium-zinc-telluride (CZT)-based SPECT system has many advantages, including better spatial and energy resolution. However, the impacts of different acquisition and reconstruction parameters on CZT SPECT quantification might still need to be validated. This study aimed to evaluate the impacts of acquisition parameters (the main energy window and acquisition time per frame) and reconstruction parameters (the number of iterations, subsets in iterative reconstruction, post-filter, and image correction methods) on the technetium quantification of CZT SPECT/CT.
\end{abstract}

Methods: A phantom (PET NEMA/IEC image quality, USA) was filled with four targetto-background (T/B) ratios (32:1, 16:1, 8:1, and 4:1) of technetium. Mean uptake values (the calculated mean concentrations for spheres) were measured to evaluate the recovery coefficient $(\mathrm{RC})$ changes under different acquisition and reconstruction parameters. The corresponding standard deviations of mean uptake values were also measured to evaluate the quantification error. Image quality was evaluated using the National Electrical Manufacturers Association (NEMA) NU 2-2012 standard.

Results: For all T/B ratios, significant correlations were found between iterations and RCs ( $r=0.62-0.96$ for $1-35$ iterations, $r=0.94-0.99$ for 35-90 iterations) as well as between the full width at half maximum (FWHM) of the Gaussian filter and RCs ( $r=-0.86$ to -1.00 , all $P$ values $<0.05$ ). The regression coefficients of $1-35$ iterations were higher than those of $35-90$ iterations (0.51-1.60 vs. 0.02-0.19). RCs calculated with AC (attenuation correction) + SC (scatter correction) + RR (resolution recovery correction) combination were more accurate (53.82-106.70\%) than those calculated with other combinations (all $P$ values $<0.05$ ). No significant statistical differences (all $P$ values $>0.05$ ) were found between the $15 \%$ and $20 \%$ energy windows except for the 32:1 T/B ratio ( $P$ value $=0.023$ ) or between the $10 \mathrm{~s} /$ frame and $120 \mathrm{~s} /$ frame acquisition times except for the 4:1 T/B ratio ( $P$ value $=0.015)$ in terms of $R C s$. party material in this article are included in the article's Creative Commons licence, unless indicated otherwise in a credit line to the material. If material is not included in the article's Creative Commons licence and your intended use is not permitted by statutory regulation or exceeds the permitted use, you will need to obtain permission directly from the copyright holder. To view a copy of this licence, visit http:// creativecommons.org/licenses/by/4.0/. 
Conclusions: CZT-SPECT/CT of technetium resulted in good quantification accuracy. The favourable acquisition parameters might be a 15\% energy window and $40 \mathrm{~s} / \mathrm{frame}$ of acquisition time. The favourable reconstruction parameters might be 35 iterations, 20 subsets, the AC + SC + RR correction combination, and no filter.

Keywords: CZT SPECT/CT, Absolute quantification, Recovery coefficient, Full width at half maximum, Attenuation correction, Scatter correction, Resolution recovery correction, OSEM

\section{Background}

Single-photon emission computed tomography (SPECT) has been widely used to diagnose various kinds of human diseases, such as myocardial diseases, endocrine disorders, and central nervous system diseases, since its invention in the 1990s [1-4]. Most of the available SPECT systems are based on the well-known Anger camera with $\mathrm{NaI}(\mathrm{Tl})$ as a scintillation material, which determines the position of an event by the centroid of the scintillation light [5]. NaI (Tl)-based detectors capture $\gamma$ photons and convert the photons into electrons, which are further amplified into strong electrical signals via photomultiplier tubes (PMTs). This conversion process introduces errors, including photon loss, motion artefacts of long acquisition time and higher radiation dosages. In recent years, digital radiographic imaging has certainly replaced analogue imaging. Digital imaging has many advantages, such as better image contrast and image enhancement [6]. In contrast to NaI SPECT, a novel digital cadmium-zinc-telluride (CZT)-based SPECT equipped with solid-state detectors generates electrical signals directly by turning incident $\gamma$ photons into electron-hole pairs under a high-voltage electric field [7]. This process avoids photon loss and produces better image quality due to its higher spatial and energy resolutions compared to those of NaI SPECT [8]. CZT SPECT also provides a shorter acquisition time and a lower radiation dosage $[9,10]$.

Absolute quantification was originally applied in positron emission computed tomography (PET). It is considered the gold standard of non-invasive quantification analysis methods for some diseases, such as coronary artery disease, microvascular disease and tumours, due to its high quantification accuracy [11, 12]. PET images display the distribution of certain radionuclides in three dimensions (3D). The data used for PET image reconstructions are in units of radioactivity per unit volume $\left(\mathrm{kBq} \cdot \mathrm{cm}^{-3}\right)$, and these data are close to the actual in vivo distribution of the radionuclide. Both PET and SPECT quantification are compromised by three major confounding variables: photon scatter, photon attenuation, and partial volume effect [13]. Scattered photons might fall into the energy window of the PET or SPECT system and therefore affect the overall quantification and image quality [14, 15]. Attenuation may lead to artefacts and inaccuracies in reconstructed images due to the highly nonuniform distribution of attenuating tissues [16]. Partial-volume effects can lead to spillover between two adjacent regions, generally resulting in the tracer uptake being underestimated. Smaller lesions often suffer severely from this effect $[17,18]$. These problems were solved and validated in PET several decades ago because of the advantages offered by positron decay and coincidence detection $[19,20]$. These problems have also subsequently been solved to some extent in SPECT. Several methods have been applied in scatter correction (SC), including the deconvolution method [21, 22], 
energy window subtraction method [23, 24], energy-weighted acquisition method $[25,26]$, inverse Monte Carlo reconstruction algorithm, and so on [27, 28]. Attenuation correction $(\mathrm{AC})$ has been achieved by using the CT-based attenuation correction method [29], Chang algorithm method [30], and so on. Additionally, some methods have been applied to reduce the partial volume effect, including image enhancement techniques [31, 32], image domain anatomically-based PVC (partial volume correction) techniques [33], projection-based PVC, and so on [34]. Despite this, both the spatial and energy resolutions of conventional $\mathrm{NaI}$ SPECT are relatively low, and radionuclides applied in SPECT have a higher fraction of scattered photons than those of PET $[35,36]$. These drawbacks might magnify the partial volume effect and compromise the effectiveness of SC and AC. As a result, SPECT images may be more difficult to quantify.

Today, however, with the development of SPECT systems, absolute quantification is also widely validated and used. Some studies have suggested that absolute SPECT quantification is promising with different SPECT equipment when reconstruction protocols are standardized [37, 38]. SPECT quantification of various radionuclides has also been well studied, including technetium-99 m $\left({ }^{99 \mathrm{~m}} \mathrm{Tc}\right)$ [39], indium-111 $\left({ }^{111} \mathrm{In}\right)$ [40], yttrium-90 $\left({ }^{90} \mathrm{Y}\right)$ [41], lutetium-177 $\left({ }^{177} \mathrm{Lu}\right)$ and so on [42]. Additionally, SPECT quantification has been widely used in clinical practice, such as quantification of the lung shunt fraction in hepatic radioembolization [43], myocardial perfusion imaging [44], monitoring cancer [45], and determining lesion volumes [46]. Despite these validations and clinical practices, various acquisition and reconstruction parameters may also affect the accuracy of SPECT quantification. Some studies have suggested that the small number of iterations and subsets used in OSEM (ordered subsets expectation maximization) reconstruction influences the quantification accuracy because of incomplete convergence $[47,48]$. The application of different correction methods, such as AC, SC, and RR (resolution recovery correction), may also affect the quantification accuracy [29, 49, 50]. However, many of these studies are based on conventional NaI SPECT systems, and therefore, the impacts of different acquisition and reconstruction parameters on absolute CZT SPECT quantification might need to be studied. This study aimed to evaluate the impacts of acquisition parameters (the main acquisition energy window and acquisition time/frame) and reconstruction parameters (the number of iterations and subsets in iterative reconstruction, post-filter, $\mathrm{AC}, \mathrm{SC}$, and $\mathrm{RR}$ correction) on the accuracy of CZT SPECT/CT technetium quantification.

\section{Materials and methods}

\section{Phantom preparation}

The phantom (NEMA/IEC 2001) used for this experiment consisted of a D-shaped cylinder and six spheres with different diameters $(37 \mathrm{~mm}, 28 \mathrm{~mm}, 22 \mathrm{~mm}, 17 \mathrm{~mm}, 13 \mathrm{~mm}$, and $10 \mathrm{~mm}$, Additional file 1: Fig. S1). We filled the phantom with ${ }^{99} \mathrm{mcO}_{4}{ }^{-}$(Atomic Technology Corporation, China) at four target-to-background (T/B) ratios (32:1, 16:1, $8: 1$, and 4:1). The radioactivity in spheres of four $\mathrm{T} / \mathrm{B}$ ratios was $0.20 \mathrm{MBq} / \mathrm{ml}(32: 1)$, $0.11 \mathrm{MBq} / \mathrm{ml}$ (16:1), $0.06 \mathrm{MBq} / \mathrm{ml}$ (8:1), and $0.03 \mathrm{MBq} / \mathrm{ml}$ (4:1), respectively, by the time of acquisition. The decay of ${ }^{99 \mathrm{~m}} \mathrm{TcO}_{4}{ }^{-}$was calibrated to the time of acquisition [51]. 


\section{Image acquisition parameters}

SPECT/CT acquisition of the PET NEMA/IEC image quality phantom was performed on a Discovery NM/CT 670 CZT (GE Healthcare, USA) equipped with wide energy high-resolution collimators. All SPECT images were acquired with a list mode. The step and shoot acquisition mode was performed by 360-degree rotations (120 s/6degree per frame) with a matrix size of $128 \times 128$ without zoom. Two main energy windows ( $140 \mathrm{keV} \pm 7.5 \%$ and $140 \mathrm{keV} \pm 10 \%)$ were reconstructed by the list mode to evaluate the impacts of the main energy window on RCs. The scatter energy window was $120 \pm 5 \% \mathrm{keV}$. CT images were acquired with a $120 \mathrm{kVp}$ tube voltage, $200 \mathrm{~mA}$ tube current, matrix size of $512 \times 512$, and $1.25 \mathrm{~mm}$ slice thickness.

\section{Image reconstruction parameters}

All images were reconstructed using the OSEM algorithm with 1-90 iterations and $2-30$ subsets [52]. The FWHM range of the Gaussian filter was $0.7-6.99 \mathrm{~mm}$. The correction methods used in this study included CT-based AC, dual-energy-window technique-based SC, and point spread function-based RR correction. Three image correction combinations were used to evaluate the impacts of the image correction methods, including $\mathrm{AC}+\mathrm{SC}+\mathrm{RR}, \mathrm{AC}+\mathrm{SC}$, and $\mathrm{AC}+\mathrm{RR}$. List mode was applied to reconstruct the acquisition time to $1-120 \mathrm{~s} /$ frame. In every step of the analysis, we evaluated the impact of a certain parameter to determine the optimal value while fixing all other parameters at the same time. All acquisition and reconstruction parameters in the evaluation process are listed in Fig. 1.

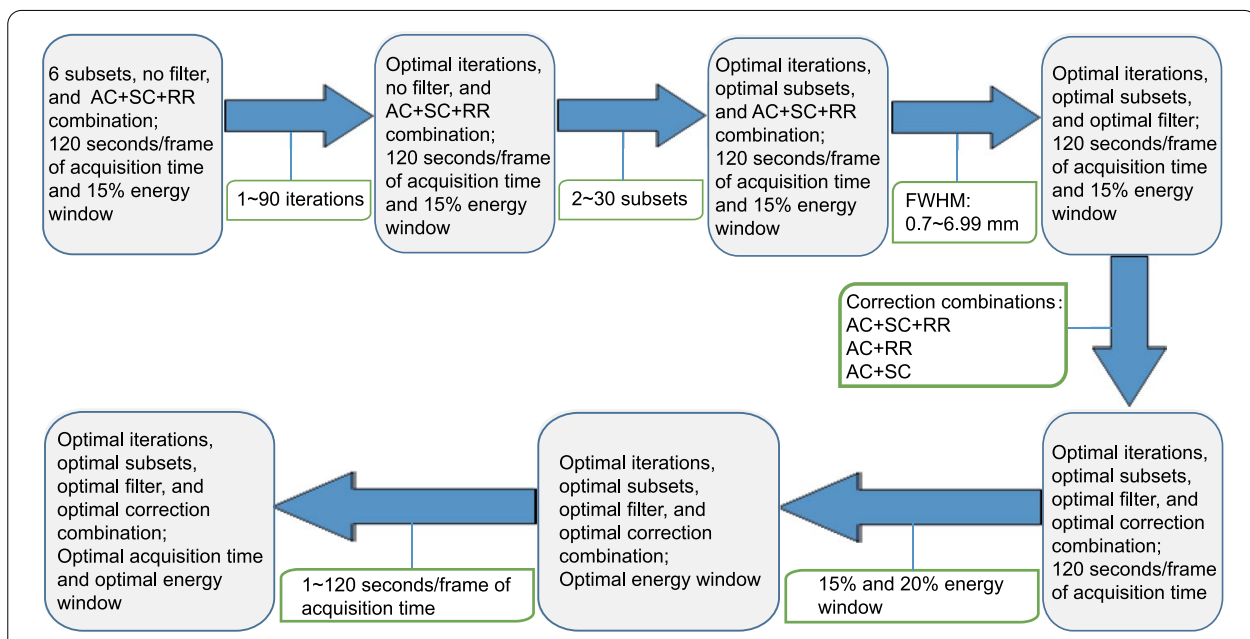

Fig. 1 Analysis process of different acquisition and reconstruction parameters. The process started with six subsets, no filter, AC+SC+RR correction combination, 120 s/frame of acquisition time, and 15\% energy window. The impacts of iterations, subsets, FWHM, correction combination, energy window, and acquisition time/frame were evaluated in sequence and an optimal value of these parameters was determined in each step of the process 


\section{Quantitative analysis}

\section{RC calculations}

Volumes of interest (VOIs) of six spheres were delineated using the inner edge of spheres of CT images as references. Mean uptake values $(\mathrm{MBq} / \mathrm{ml})$ and corresponding standard deviations (SDs) were automatically calculated three times by the Q. Metrix of GE-Xeleris 4.0 workstation (GE Healthcare, USA) and are shown as averages. RCs were calculated using Eq. (1) [19]:

$$
R C=\frac{\text { Mean measured radioactivity concentration }}{\text { Actual radioactivity concentration }} \times 100 \%
$$

\section{Image quality evaluations}

To assess the image quality, we calculated both the per cent contrast and coefficient of variation (COV) complying with the NEMA NU 2-2012 standard [53-56]. The per cent contrast $Q_{H, j}$ for each hot sphere was calculated by using Eq. (2):

$$
Q_{\mathrm{H}, \mathrm{j}}=\frac{C_{\mathrm{H}, \mathrm{j}} / C_{\mathrm{B}, \mathrm{j}}-1}{a_{\mathrm{H}} / a_{\mathrm{B}}-1} \times 100 \% .
$$

where $C_{\mathrm{H}, j}$ is the average count in the region of interest (ROI) for sphere $j, C_{\mathrm{B}, j}$ is the average of the background ROI counts for sphere $j, a_{\mathrm{H}}$ is the activity concentration in the hot spheres, and $a_{\mathrm{B}}$ is the activity concentration in the background.

The COV $N_{j}$ for each hot sphere was calculated by using Eq. (3):

$$
N_{j}=\frac{\mathrm{SD}_{j}}{C_{\mathrm{B}, \mathrm{j}}} \times 100 \%
$$

where $\mathrm{SD}_{j}$ is the standard deviation of the background ROI counts for sphere $j$.

\section{Statistical analysis}

All statistical analyses were performed by SPSS 23.0 (IBM, USA). All graphs were produced by GraphPad Prism 8.3.0 (GraphPad Software, USA) and Origin Pro 2021 (OriginLab, USA). The relationships between RCs and the different number of iterations and subsets, FWHM, and acquisition time/frame were established by Pearson's rank correlation and linear regression analysis. RCs and per cent contrast of three different correction combinations were compared using the paired t-test [57]. The comparison of RCs for different energy windows and acquisition time/frame was also analysed by using the paired t-test. A $P$ value lower than 0.05 was considered statistically significant.

\section{Results}

Impacts of the number of iterations and subsets

Figure 2 shows that of all the T/B ratios, the RCs of larger spheres converged earlier than those of smaller spheres, of which $37-17 \mathrm{~mm}$ spheres converged at 35 iterations and 13-10 mm spheres converged at 85 iterations. Table 1 shows that apart from the $37 \mathrm{~mm}$ sphere of $1-35$ iterations of the $32: 1$ and 16:1 T/B ratios, there were significant positive 


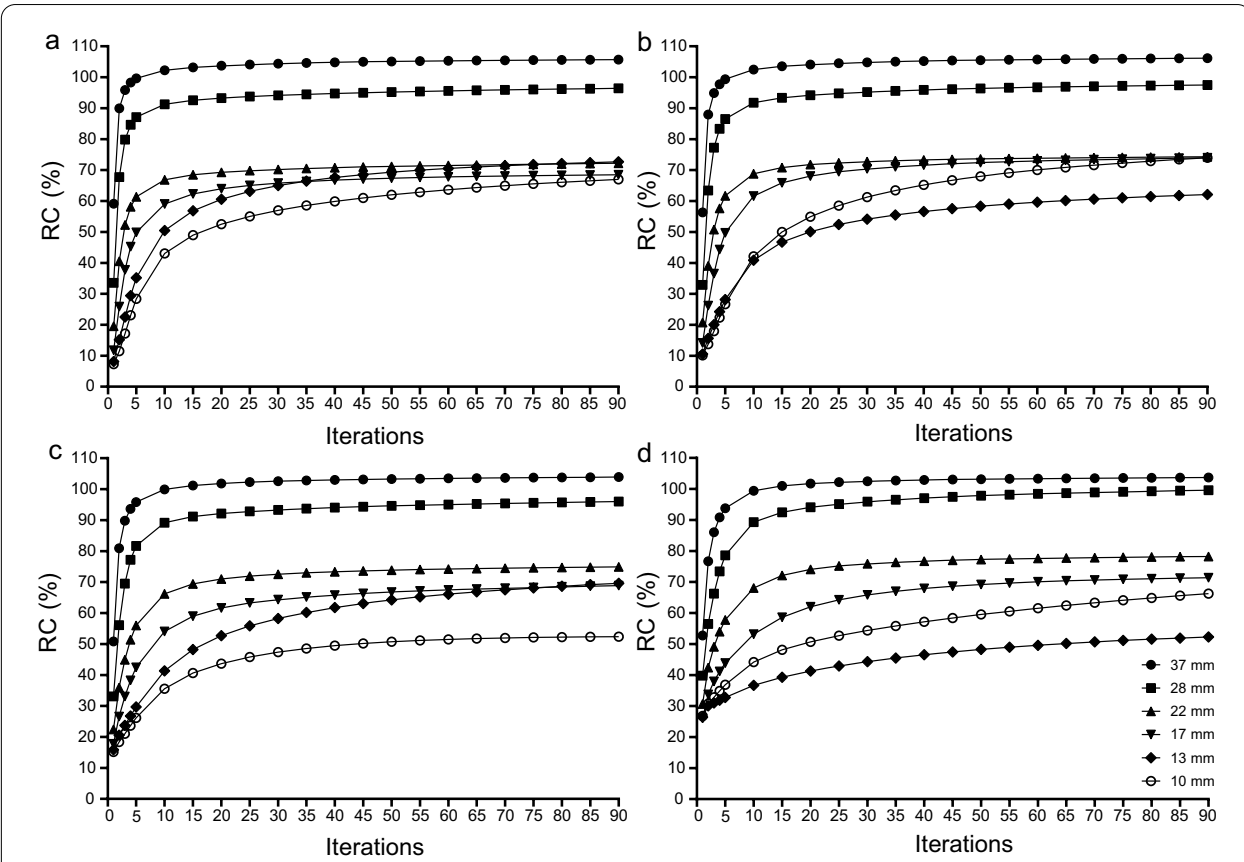

Fig. 2 Impacts of iterations on $\mathrm{RCs}$. a 32:1 T/B ratio; b 16:1 T/B ratio; c 8:1 T/B ratio; $\mathbf{d}$ 4:1 T/B ratio; $R C$ s were calculated with 1-90 iterations; Fixed reconstruction parameters: six subsets, no filter, AC + SC+RR combination; Fixed acquisition parameters: 120 s/frame of acquisition time, 15\% energy window

correlations between RCs and iterations $(r=0.62-0.96$ for $1-35$ iterations, $r=0.94-0.99$ for 35-90 iterations, all $P$ values $<0.05)$. Linear regression analysis shows that $1-35$ iterations had much higher regression coefficients than those of 35-90 iterations $(0.63-1.60$ vs. $0.02-0.15$ for the $32: 1 \mathrm{~T} / \mathrm{B}$ ratio, $0.70-1.59$ vs. $0.02-0.15$ for the $16: 1 \mathrm{~T} / \mathrm{B}$ ratio, $0.80-1.29$ vs. $0.02-0.17$ for the $8: 1 \mathrm{~T} / \mathrm{B}$ ratio, and $0.51-1.26$ vs. $0.02-0.19$ for the $4: 1 \mathrm{~T} / \mathrm{B}$ ratio). RCs increased rapidly within the first 35 iterations.

Figure 3 shows that RCs did not increase rapidly with an increasing number of subsets. RCs of larger spheres $(37-17 \mathrm{~mm}$ ) became stable after 20 subsets. Table 1 shows that the Pearson $r$ values of the six spheres ranged from 0.68 to 0.89 (32:1 T/B ratio), 0.61 to 0.90 (16:1 T/B ratio), 0.52 to 0.85 (8:1 T/B ratio), and -0.34 to 0.98 (4:1 T/B ratio). In linear regression analysis, the regression coefficients of the six spheres ranged from 0.09 to 0.70 for the $32: 1 \mathrm{~T} / \mathrm{B}$ ratio, 0.07 to 1.14 for the $16: 1 \mathrm{~T} / \mathrm{B}$ ratio, 0.08 to 0.90 for the $8: 1 \mathrm{~T} / \mathrm{B}$ ratio, and -0.06 to 1.14 for the $4: 1 \mathrm{~T} / \mathrm{B}$ ratio.

\section{Impacts of the Gaussian filter}

In Fig. 4, RCs of all spheres declined significantly as the FWHM $(0.7-6.99 \mathrm{~mm})$ of the Gaussian filter increased. There were significant negative correlations between the FWHM of the Gaussian filter and RCs for all spheres $(r:-0.87$ to -1.00 for the $32: 1 \mathrm{~T} / \mathrm{B}$ ratio, -0.86 to -1.00 for the $16: 1 \mathrm{~T} / \mathrm{B}$ ratio, -0.90 to -1.00 for the $8: 1 \mathrm{~T} / \mathrm{B}$ ratio, and -0.89 to -1.00 for the $4: 1 \mathrm{~T} / \mathrm{B}$ ratio, all $P$ values $<0.05)$. Additionally, there were high regression coefficients in all 6 spheres $(-9.49$ to -11.83 for the $32: 1 \mathrm{~T} / \mathrm{B}$ ratio, -8.68 to -11.83 for the $16: 1 \mathrm{~T} / \mathrm{B}$ ratio, -6.23 to -10.90 for the $8: 1 \mathrm{~T} / \mathrm{B}$ ratio, and -4.23 
Table 1 Correlation and linear regression analysis of RCs among different acquisition and reconstruction parameters

\begin{tabular}{|c|c|c|c|c|c|c|c|c|}
\hline & & & $37 \mathrm{~mm}$ & $28 \mathrm{~mm}$ & $22 \mathrm{~mm}$ & $17 \mathrm{~mm}$ & $13 \mathrm{~mm}$ & $10 \mathrm{~mm}$ \\
\hline \multirow[t]{10}{*}{$32: 1$} & Iterations (1-35) & $\begin{array}{l}\text { Regression coef- } \\
\text { ficient }\end{array}$ & 0.63 & 0.93 & 0.93 & 1.21 & 1.60 & 1.47 \\
\hline & & r & 0.58 & $0.62^{*}$ & $0.70^{*}$ & $0.80^{*}$ & $0.90^{* *}$ & $0.92^{* *}$ \\
\hline & Iterations (35-90) & $\begin{array}{l}\text { Regression coef- } \\
\text { ficient }\end{array}$ & 0.02 & 0.03 & 0.03 & 0.04 & 0.11 & 0.15 \\
\hline & & $r$ & $0.98^{* *}$ & $0.99^{* *}$ & $0.99^{* *}$ & $0.97^{* *}$ & $0.97^{* *}$ & $0.99^{* *}$ \\
\hline & Subsets & $\begin{array}{l}\text { Regression coef- } \\
\text { ficient }\end{array}$ & 0.09 & 0.15 & 0.20 & 0.39 & 0.69 & 0.70 \\
\hline & & r & 0.68 & $0.80^{*}$ & $0.87^{*}$ & $0.89^{*}$ & $0.84^{*}$ & $0.85^{*}$ \\
\hline & FWHM & $\begin{array}{l}\text { Regression coef- } \\
\text { ficient }\end{array}$ & -11.36 & -11.83 & -9.96 & -10.28 & -10.88 & -9.49 \\
\hline & & r & $-1.00^{* *}$ & $-0.99^{* *}$ & $-0.98^{* *}$ & $-0.96^{* *}$ & $-0.92^{*}$ & $-0.87^{*}$ \\
\hline & $\begin{array}{l}\text { Acquisition time (s/ } \\
\text { frame) }\end{array}$ & $\begin{array}{l}\text { Regression coef- } \\
\text { ficient }\end{array}$ & -0.01 & 0.04 & 0.07 & 0.05 & 0.05 & 0.05 \\
\hline & & r & -0.62 & $0.84^{*}$ & $0.72^{*}$ & 0.66 & 0.36 & 0.36 \\
\hline \multirow[t]{10}{*}{$16: 1$} & Iterations (1-35) & $\begin{array}{l}\text { Regressioncoef- } \\
\text { ficient }\end{array}$ & 0.70 & 1.04 & 1.03 & 1.37 & 1.28 & 1.59 \\
\hline & & r & 0.59 & $0.66^{*}$ & $0.74^{*}$ & $0.84^{*}$ & $0.92^{* *}$ & $0.95^{* *}$ \\
\hline & Iterations (35-90) & $\begin{array}{l}\text { Regression coef- } \\
\text { ficient }\end{array}$ & 0.02 & 0.03 & 0.03 & 0.04 & 0.11 & 0.15 \\
\hline & & $r$ & $0.98^{* *}$ & $0.98^{* *}$ & $0.98^{* *}$ & $0.98^{* *}$ & $0.98^{* *}$ & $0.98^{* *}$ \\
\hline & Subsets & $\begin{array}{l}\text { Regression coef- } \\
\text { ficient }\end{array}$ & 0.07 & 0.12 & 0.13 & 0.35 & 0.69 & 1.14 \\
\hline & & $r$ & 0.61 & 0.64 & 0.70 & $0.76^{*}$ & $0.86^{*}$ & $0.90^{*}$ \\
\hline & FWHM & $\begin{array}{l}\text { Regression coef- } \\
\text { ficient }\end{array}$ & -11.02 & -11.83 & -9.85 & -10.65 & -8.68 & -10.09 \\
\hline & & r & $-1.00^{* *}$ & $-0.99^{* *}$ & $-0.99^{* *}$ & $-0.96^{* *}$ & $-0.92^{*}$ & $-0.86^{*}$ \\
\hline & $\begin{array}{l}\text { Acquisition time (s/ } \\
\text { frame) }\end{array}$ & $\begin{array}{l}\text { Regression coef- } \\
\text { ficient }\end{array}$ & -0.04 & -0.03 & -0.02 & -0.04 & 0.02 & 0.28 \\
\hline & & r & -0.63 & -0.55 & $-0.73^{*}$ & -0.68 & 0.29 & $0.82^{*}$ \\
\hline \multirow[t]{10}{*}{$8: 1$} & Iterations (1-35) & $\begin{array}{l}\text { Regression coef- } \\
\text { ficient }\end{array}$ & 0.80 & 1.15 & 1.15 & 1.23 & 1.29 & 0.98 \\
\hline & & r & $0.63^{*}$ & $0.72^{*}$ & $0.82^{*}$ & $0.88^{* *}$ & $0.95^{* *}$ & $0.94^{* *}$ \\
\hline & Iterations (35-90) & $\begin{array}{l}\text { Regression coef- } \\
\text { ficient }\end{array}$ & 0.02 & 0.04 & 0.03 & 0.06 & 0.17 & 0.06 \\
\hline & & r & $0.98^{* *}$ & $0.99^{* *}$ & $0.98^{* *}$ & $0.98^{* *}$ & $0.98^{* *}$ & $0.94^{* *}$ \\
\hline & Subsets & $\begin{array}{l}\text { Regression coef- } \\
\text { ficient }\end{array}$ & 0.08 & 0.10 & 0.26 & 0.47 & 0.90 & 0.40 \\
\hline & & r & 0.65 & 0.52 & $0.83^{*}$ & $0.85^{*}$ & $0.83^{*}$ & 0.66 \\
\hline & FWHM & $\begin{array}{l}\text { Regression coef- } \\
\text { ficient }\end{array}$ & -9.95 & -10.90 & -8.73 & -8.79 & -9.22 & -6.23 \\
\hline & & $r$ & $-1.00^{* *}$ & $-0.99^{* *}$ & $-0.98^{* *}$ & $-0.96^{* *}$ & $-0.92^{*}$ & $-0.90^{*}$ \\
\hline & $\begin{array}{l}\text { Acquisition time (s/ } \\
\text { frame) }\end{array}$ & $\begin{array}{l}\text { Regression coef- } \\
\text { ficient }\end{array}$ & 0.09 & 0.04 & -0.02 & 0.01 & 0.12 & -0.31 \\
\hline & & r & 0.70 & 0.62 & -0.44 & 0.02 & 0.43 & $-0.71^{*}$ \\
\hline \multirow[t]{5}{*}{$4: 1$} & Iterations (1-35) & $\begin{array}{l}\text { Regression coef- } \\
\text { ficient }\end{array}$ & 0.87 & 1.26 & 1.10 & 1.09 & 0.51 & 0.81 \\
\hline & & r & $0.69^{*}$ & $0.81^{*}$ & $0.85^{* *}$ & $0.92^{* *}$ & $0.96^{* *}$ & $0.95^{* *}$ \\
\hline & Iterations (35-90) & $\begin{array}{l}\text { Regression coef- } \\
\text { ficient }\end{array}$ & 0.02 & 0.05 & 0.03 & 0.07 & 0.12 & 0.19 \\
\hline & & $r$ & $0.98^{* *}$ & $0.98^{* *}$ & $0.97^{* *}$ & $0.96^{* *}$ & $0.99^{* *}$ & $0.99^{* *}$ \\
\hline & Subsets & $\begin{array}{l}\text { Regression coef- } \\
\text { ficient }\end{array}$ & -0.06 & 0.11 & 0.16 & 0.50 & 0.56 & 1.14 \\
\hline
\end{tabular}


Table 1 (continued)

\begin{tabular}{llrrrrrr}
\hline & & $\mathbf{3 7} \mathbf{~ m m}$ & $\mathbf{2 8} \mathbf{~ m m}$ & $\mathbf{2 2} \mathbf{~ m m}$ & $\mathbf{1 7} \mathbf{~ m m}$ & $\mathbf{1 3} \mathbf{~ m m}$ & $\mathbf{1 0 ~} \mathbf{~ m m}$ \\
\hline \multirow{3}{*}{ FWHM } & $r$ & -0.34 & 0.44 & 0.57 & $0.76^{*}$ & $0.87^{*}$ & $0.98^{* *}$ \\
& $\begin{array}{l}\text { Regression coef- } \\
\text { ficient }\end{array}$ & -8.59 & -9.39 & -7.24 & -7.51 & -4.23 & -6.10 \\
& $r$ & $-1.00^{* *}$ & $-0.99^{* *}$ & $-0.99^{* *}$ & $-0.96^{* *}$ & $-0.90^{*}$ & $-0.89^{*}$ \\
$\begin{array}{l}\text { Acquisition time } \\
\text { frame) }\end{array}$ & $\begin{array}{l}\text { Regression coef- } \\
\text { ficient }\end{array}$ & 0.16 & 0.21 & -0.08 & 0.24 & 0.26 & -0.34 \\
& $r$ & $0.71^{*}$ & $0.76^{*}$ & -0.68 & $0.82^{*}$ & 0.70 & -0.53 \\
\hline
\end{tabular}

${ }^{*}$,,$P$-value $<0.001 ; *, P$-value $<0.05 ; r$, Pearson $r$

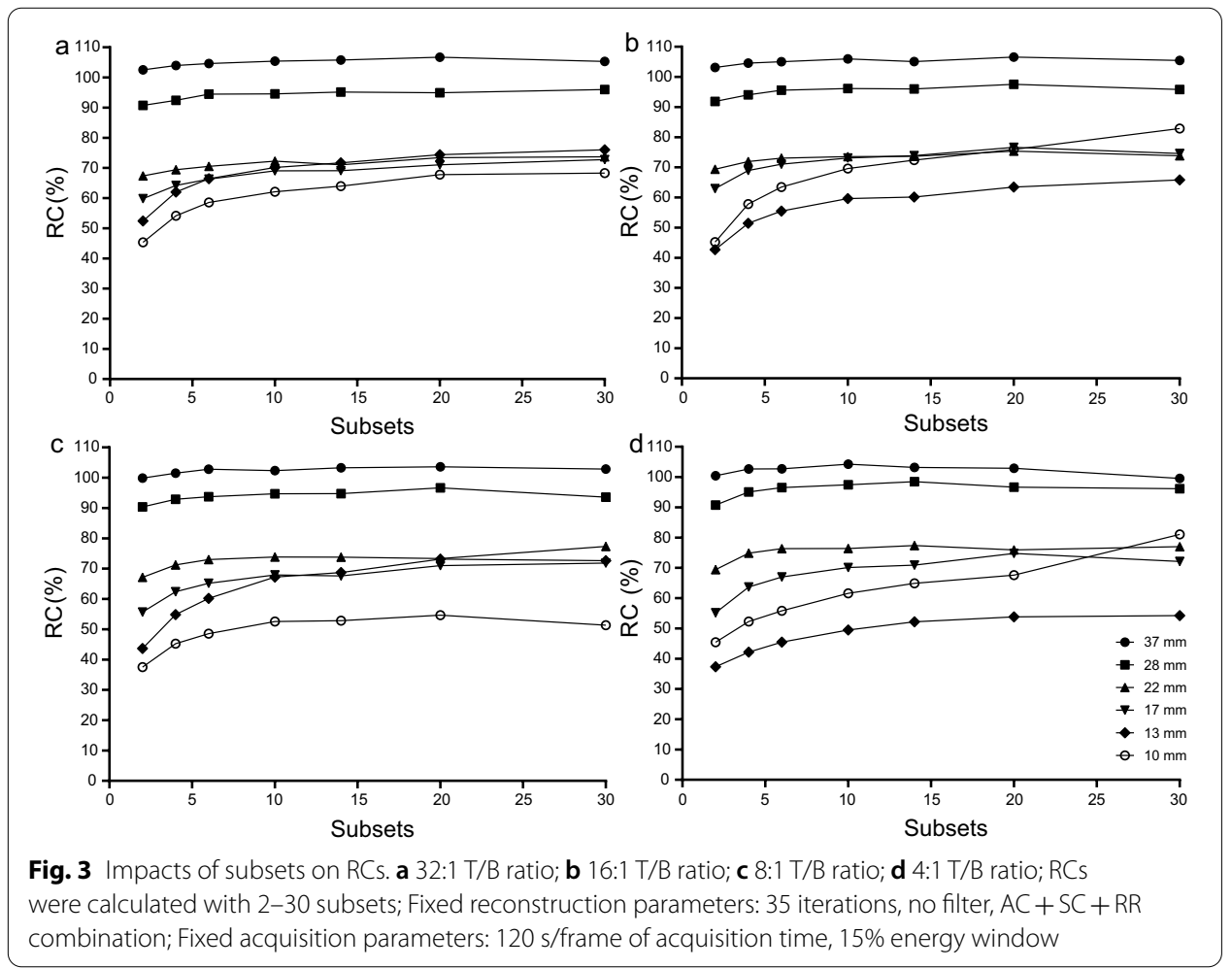

to -9.39 for the $4: 1 \mathrm{~T} / \mathrm{B}$ ratio, Table 1 ). Furthermore, there was no FWHM plateau in terms of RCs, which was different from iterations or subsets.

\section{Impacts of the image correction methods}

The profiles of four T/B ratios show that $\mathrm{RCs}$ of the $\mathrm{AC}+\mathrm{SC}+\mathrm{RR}$ correction combination were closer to the actual sphere activity concentration. The $A C+R R$ combination predicted the highest mean uptake values, while the $\mathrm{AC}+\mathrm{SC}$ combination predicted the lowest mean uptake values in spheres (Fig. 5). Table 2 shows that the RCs of the $\mathrm{AC}+\mathrm{SC}+\mathrm{RR}$ combination were lower than those of the $\mathrm{AC}+\mathrm{RR}$ combination but higher than those of the AC+SC combination (67.80-106.70\% vs. $75.68-120.23 \%$ vs. 29.91-67.96\% for the $32: 1 \mathrm{~T} / \mathrm{B}$ ratio; $63.44-106.57 \%$ vs. $72.80-122.00 \%$ vs. $30.89-71.98 \%$ for the $16: 1 \mathrm{~T} / \mathrm{B}$ ratio; $54.67-103.58 \%$ vs. $62.93-120.84 \%$ vs. $28.21-71.91 \%$ for the 

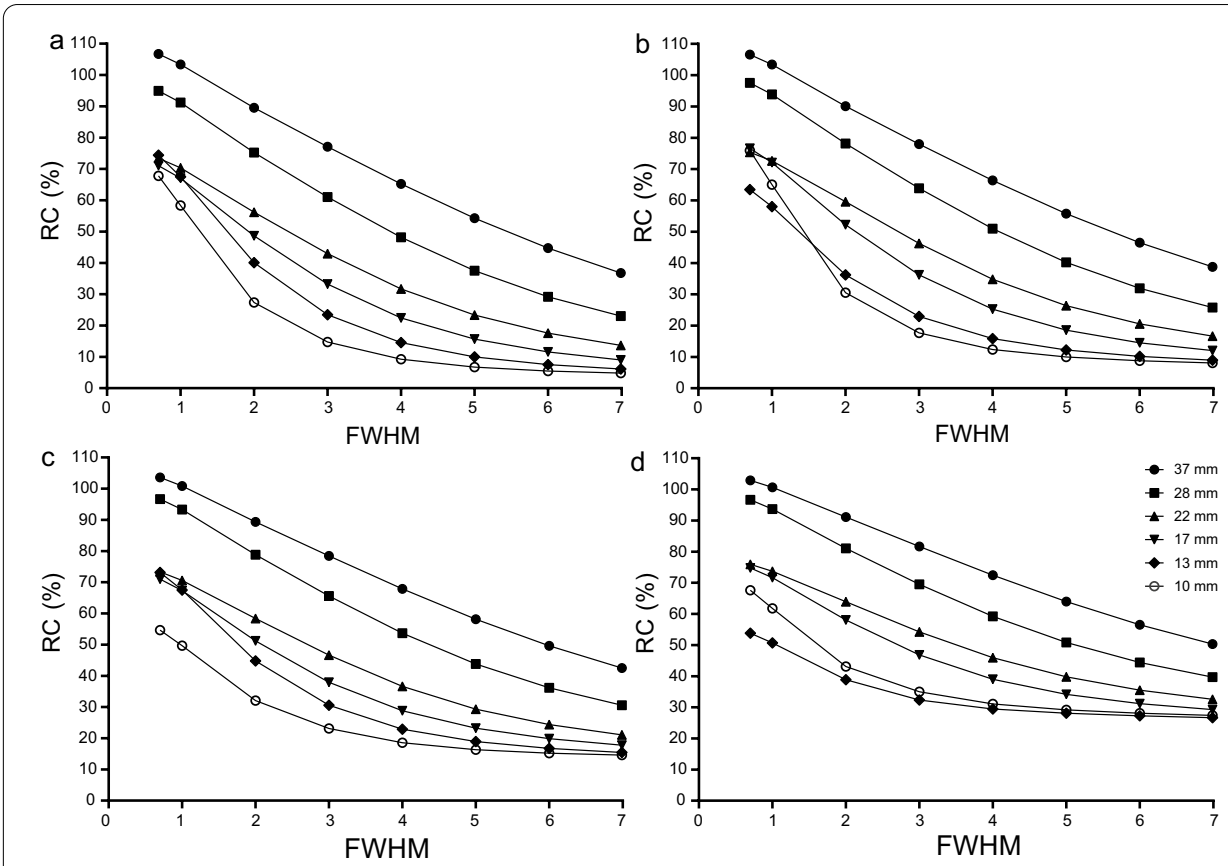

Fig. 4 Impacts of FWHM value of the Gaussian filter on RCs. a 32:1 T/B ratio; b 16:1 T/B ratio; c 8:1 T/B ratio; d 4:1 T/B ratio; RCs were calculated with FWHM value of 0.70-6.99; Fixed reconstruction parameters: 35 iterations, 20 subsets, $A C+S C+R R$ combination; Fixed acquisition parameters: 120 s/frame of acquisition time, $15 \%$ energy window
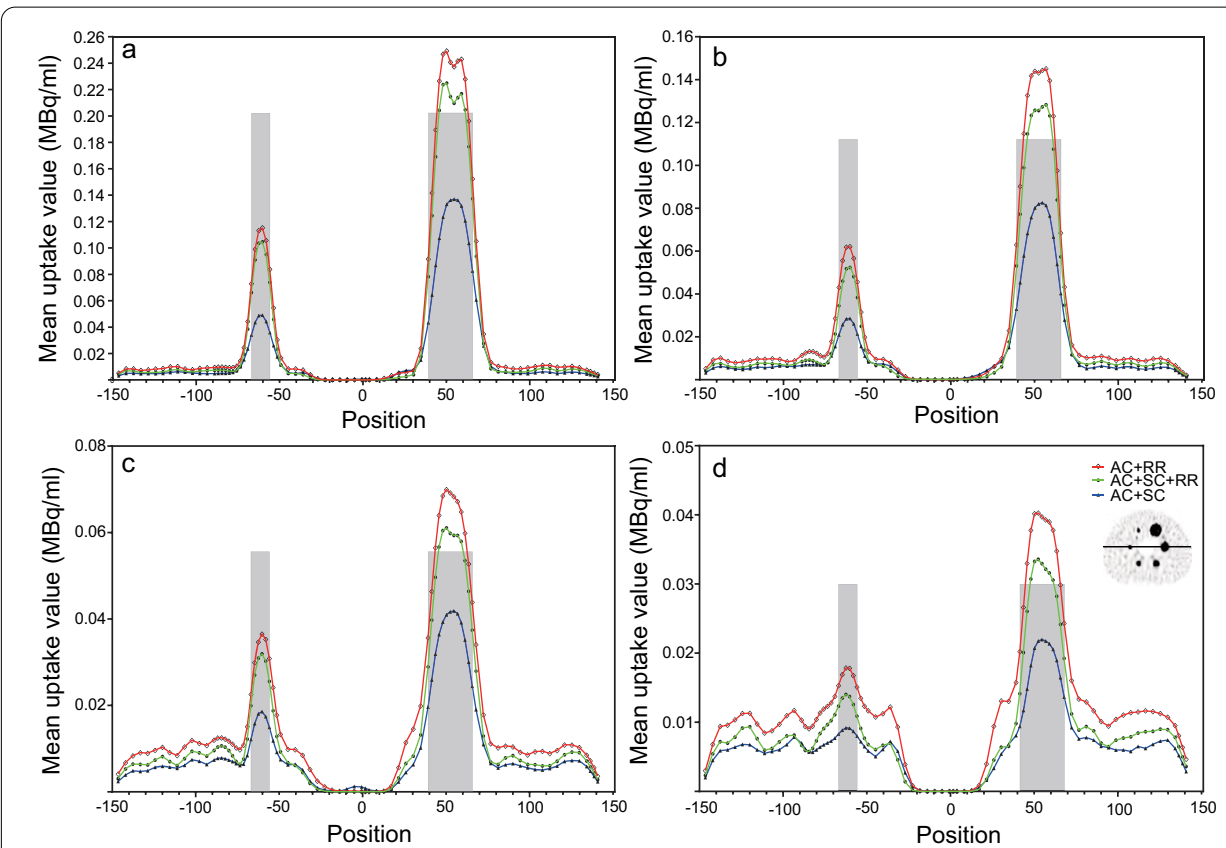

Fig. 5 Location of line profiles of 32:1 (a), 16:1 (b), 8:1 (c), and 4:1 (d) T/B ratios through the central slice of the entire phantom. $X$-axis indicates the pixel position of the phantom; $Y$-axis indicates the calculated mean uptake values of the phantom; The upper edges of both grey areas are the actual radioactivity concentration in spheres; Different curves were calculated mean uptake values by using different correction combinations $(\mathrm{AC}+\mathrm{SC}+\mathrm{RR}, \mathrm{AC}+\mathrm{SC}$, and $\mathrm{AC}+\mathrm{RR}) ;$ Fixed reconstruction parameters: 35 iterations, 20 subsets, no filter; Fixed acquisition parameters: $120 \mathrm{~s} /$ frame of acquisition time, 15\% energy window 
Table 2 Comparison of RCs (\%) of all spheres among three different correction combinations

\begin{tabular}{|c|c|c|c|c|c|c|c|c|}
\hline & Correction combination & $37 \mathrm{~mm}$ & $28 \mathrm{~mm}$ & $22 \mathrm{~mm}$ & $17 \mathrm{~mm}$ & $13 \mathrm{~mm}$ & $10 \mathrm{~mm}$ & $P$ value \\
\hline \multirow[t]{3}{*}{$32: 1$} & $\mathrm{AC}+\mathrm{SC}+\mathrm{RR}$ & 106.70 & 94.94 & 73.44 & 71.06 & 74.39 & 67.80 & \\
\hline & $A C+R R$ & 120.23 & 107.38 & 82.77 & 78.93 & 80.52 & 75.68 & $<0.001^{\mathrm{a}}$ \\
\hline & $A C+S C$ & 67.96 & 53.86 & 41.65 & 34.67 & 29.91 & 31.02 & $<0.001^{b}$ \\
\hline \multirow[t]{3}{*}{$16: 1$} & $A C+S C+R R$ & 106.57 & 97.53 & 75.37 & 76.64 & 63.44 & 75.91 & \\
\hline & $A C+R R$ & 122.00 & 109.81 & 84.78 & 87.25 & 72.80 & 81.33 & $<0.001^{\mathrm{a}}$ \\
\hline & $A C+S C$ & 71.98 & 59.22 & 43.23 & 41.91 & 30.89 & 32.54 & $<0.001^{b}$ \\
\hline \multirow[t]{3}{*}{$8: 1$} & $A C+S C+R R$ & 103.58 & 96.65 & 73.31 & 70.98 & 73.14 & 54.67 & \\
\hline & $A C+R R$ & 120.84 & 112.18 & 88.17 & 84.09 & 81.65 & 62.93 & $<0.001^{\mathrm{a}}$ \\
\hline & $A C+S C$ & 71.91 & 60.81 & 45.30 & 41.41 & 38.88 & 28.21 & $<0.001^{b}$ \\
\hline \multirow[t]{3}{*}{$4: 1$} & $\mathrm{AC}+\mathrm{SC}+\mathrm{RR}$ & 102.89 & 96.64 & 75.93 & 74.79 & 53.82 & 67.59 & \\
\hline & $A C+R R$ & 123.41 & 115.88 & 92.70 & 88.94 & 66.64 & 75.21 & $<0.001^{\mathrm{a}}$ \\
\hline & $A C+S C$ & 73.86 & 62.46 & 51.10 & 44.19 & 34.34 & 39.69 & $<0.001^{b}$ \\
\hline
\end{tabular}

Reconstruction parameters: 35 iterations, 20 subsets, no filter; Acquisition parameters: $120 \mathrm{~s} /$ frame of acquisition time, 15\% main energy window; $A C$, attenuation correction; $S C$, scatter correction; $R R$, resolution recovery correction; $a, A C+S C+R R$ vs. $A C+R R ; b, A C+S C+R R$ vs. $A C+S C$

8:1 T/B ratio; $53.82-102.89 \%$ vs. $66.64-123.41 \%$ vs. $34.34-73.86 \%$ for the $4: 1 \mathrm{~T} / \mathrm{B}$ ratio, all $P$ values $<0.05)$. Figure 6 shows the visual difference of the images reconstructed with different correction combinations. Among all T/B ratios, the AC+SC+RR combination had a better visual image quality. Table 3 shows that the per cent contrasts of six spheres reconstructed using the $\mathrm{AC}+\mathrm{SC}+\mathrm{RR}$ combination were higher than those of other correction combinations $(\mathrm{AC}+\mathrm{SC}+\mathrm{RR}$ vs. $\mathrm{AC}+\mathrm{RR}$; $\mathrm{AC}+\mathrm{SC}+\mathrm{RR}$ vs. $\mathrm{AC}+\mathrm{SC}$, all $P$ values $<0.05)$. However, the COVs of the $A C+R R$ combination were lower than those of the $\mathrm{AC}+\mathrm{SC}+\mathrm{RR}$ combination or $\mathrm{AC}+\mathrm{RR}$ combination (all $P$ values $<0.05$ ). The COVs of the $\mathrm{AC}+\mathrm{SC}$ combination were higher than those of the $\mathrm{AC}+\mathrm{SC}+\mathrm{RR}$ combination (100.70-103.52\% vs. $85.95-93.77 \%$ for the 32:1 T/B ratio, $94.43-97.64 \%$ vs. $85.71-95.09 \%$ for the $16: 1 \mathrm{~T} / \mathrm{B}$ ratio, $93.75-96.31 \%$ vs. $87.10-97.25 \%$ for the $8: 1 \mathrm{~T} / \mathrm{B}$ ratio, $91.38-94.04 \%$ vs. $79.55-92.71 \%$ for the $4: 1 \mathrm{~T} / \mathrm{B}$ ratio, $P$ values of $32: 1,16: 1$, and 4:1 T/B ratios $<0.05$, Table 4).

\section{Impacts of the main energy window}

As shown in Table 5, for the 32:1 T/B ratio, RCs under the $15 \%$ energy window were higher than those under the $20 \%$ energy window, and there was a statistically significant difference $(P$ value $=0.023)$. However, for lower T/B ratios $(16: 1,8: 1$, and 4:1), there were no statistically significant differences (all $P$ values $>0.05$ ). Since a $15 \%$ energy window might improve the quantification accuracy for a higher T/B ratio (32:1), it was determined to be the optimal energy window in this step.

\section{Impacts of the acquisition time per frame}

The correlations between RCs and the acquisition time/frame were -0.62 to 0.84 for the $32: 1 \mathrm{~T} / \mathrm{B}$ ratio, -0.73 to 0.82 for the $16: 1 \mathrm{~T} / \mathrm{B}$ ratio, -0.71 to 0.70 for the $8: 1 \mathrm{~T} / \mathrm{B}$ ratio, and -0.68 to 0.82 for the $4: 1 \mathrm{~T} / \mathrm{B}$ ratio. Regression coefficients between $\mathrm{RCs}$ and the acquisition time/frame were -0.01 to 0.07 for the $32: 1 \mathrm{~T} / \mathrm{B}$ ratio, -0.04 to 0.28 for the $16: 1 \mathrm{~T} / \mathrm{B}$ ratio, -0.31 to 0.12 for the $8: 1 \mathrm{~T} / \mathrm{B}$ ratio, and -0.34 to 0.26 for the 


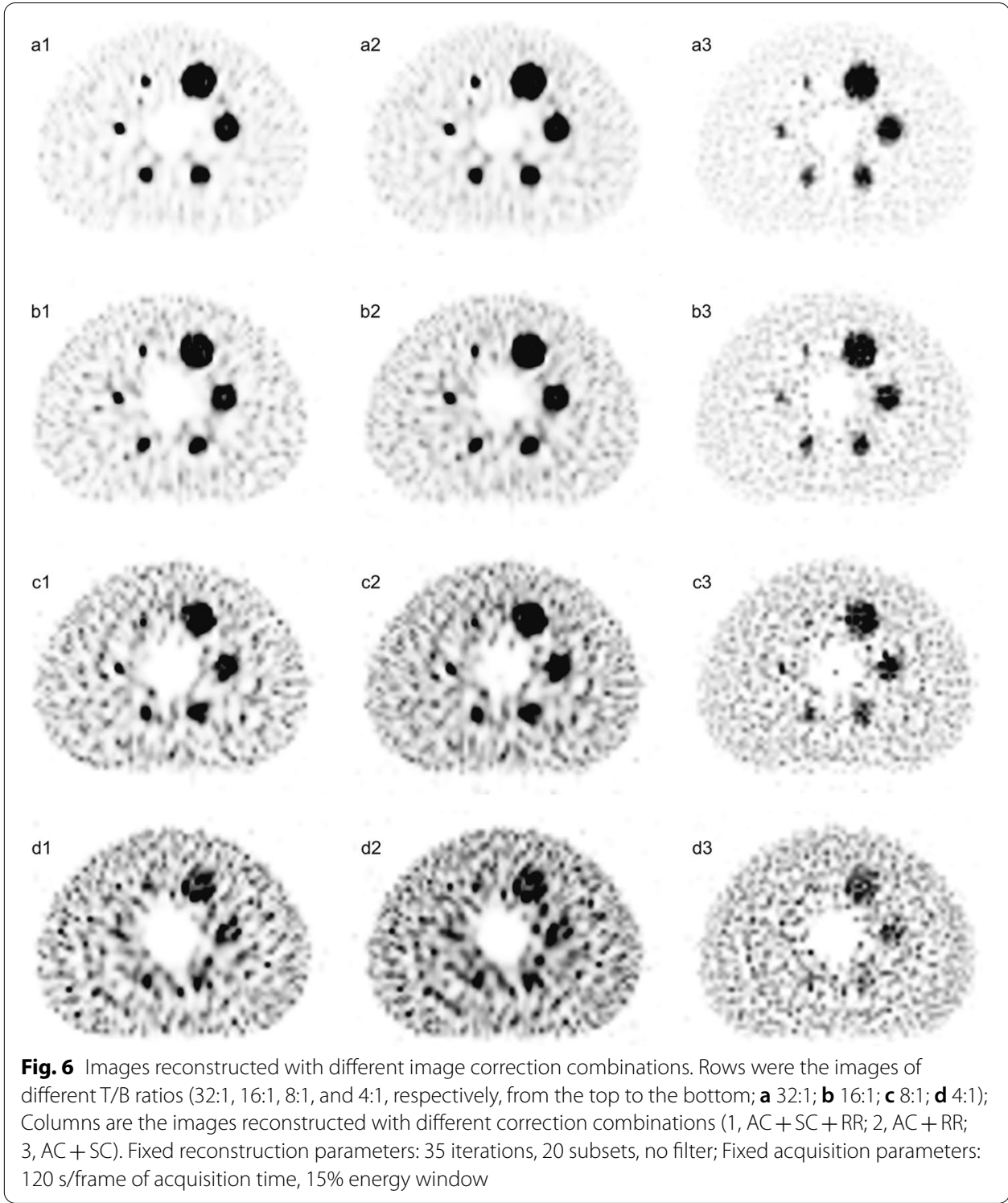

4:1 T/B ratio (Table 1). Figure 7 shows that for 32:1, $16: 1$ and $8: 1 \mathrm{~T} / \mathrm{B}$ ratios, RCs did not increase significantly with increasing acquisition time/frame (all $P$ values $>0.05$ when comparing $10 \mathrm{~s}$ with $120 \mathrm{~s} /$ frame of acquisition time). However, for the 4:1 T/B ratio, the RCs of six spheres increased significantly within the first $40 \mathrm{~s} /$ frame of acquisition time $(10 \mathrm{~s}$ vs. $120 \mathrm{~s}, P$ value $=0.015 ; 40 \mathrm{~s}$ vs. $120 \mathrm{~s}, P$ value $=0.060)$. Figure 8 shows that for most spheres of all T/B ratios, the $\mathrm{SD}$ of the mean uptake values decreased significantly in the first $40 \mathrm{~s} /$ frame of acquisition time and then remained stable. Table 6 shows that the RCs were $71.78-107.65 \%$ for the $32: 1 \mathrm{~T} / \mathrm{B}$ ratio, 58.92 $104.55 \%$ for the $16: 1 \mathrm{~T} / \mathrm{B}$ ratio, $56.57-104.44 \%$ for the $8: 1 \mathrm{~T} / \mathrm{B}$ ratio, $29.80-102.02 \%$ for the 4:1 T/B ratio under the optimal $40 \mathrm{~s} /$ frame of acquisition time, and other optimal reconstruction and acquisition parameters as follows: 35 iterations, 20 subsets, no filter, $\mathrm{AC}+\mathrm{SC}+\mathrm{RR}$ combination and $15 \%$ energy window. 
Table 3 Comparison of per cent contrasts (\%) of all spheres among three different correction combinations

\begin{tabular}{|c|c|c|c|c|c|c|c|c|}
\hline & Correction combination & $37 \mathrm{~mm}$ & $28 \mathrm{~mm}$ & $22 \mathrm{~mm}$ & $17 \mathrm{~mm}$ & $13 \mathrm{~mm}$ & $10 \mathrm{~mm}$ & $P$ value \\
\hline \multirow[t]{3}{*}{$32: 1$} & $\mathrm{AC}+\mathrm{SC}+\mathrm{RR}$ & 88.95 & 80.36 & 77.35 & 71.82 & 59.65 & 54.13 & \\
\hline & $A C+R R$ & 73.37 & 65.86 & 64.64 & 56.71 & 46.45 & 43.12 & $<0.001^{\mathrm{a}}$ \\
\hline & $\mathrm{AC}+\mathrm{SC}$ & 58.30 & 42.81 & 32.41 & 15.69 & 8.91 & 7.41 & $<0.001^{b}$ \\
\hline \multirow[t]{3}{*}{$16: 1$} & $\mathrm{AC}+\mathrm{SC}+\mathrm{RR}$ & 92.82 & 79.25 & 85.58 & 62.21 & 59.91 & 48.74 & \\
\hline & $A C+R R$ & 76.85 & 64.98 & 71.41 & 51.78 & 51.15 & 37.85 & $<0.001^{\mathrm{a}}$ \\
\hline & $A C+S C$ & 80.79 & 66.69 & 60.76 & 43.97 & 28.20 & 23.83 & $0.001^{b}$ \\
\hline \multirow[t]{3}{*}{$8: 1$} & $A C+S C+R R$ & 78.65 & 71.05 & 64.37 & 57.63 & 43.49 & 39.34 & \\
\hline & $A C+R R$ & 69.73 & 65.77 & 50.55 & 52.58 & 37.50 & 32.00 & $0.002^{\mathrm{a}}$ \\
\hline & $A C+S C$ & 77.93 & 63.92 & 38.94 & 38.93 & 28.40 & 22.11 & $0.011^{\mathrm{b}}$ \\
\hline \multirow[t]{3}{*}{$4: 1$} & $\mathrm{AC}+\mathrm{SC}+\mathrm{RR}$ & 99.94 & 76.67 & 72.83 & 66.09 & 45.03 & 65.30 & \\
\hline & $A C+R R$ & 80.81 & 62.67 & 64.85 & 51.79 & 39.67 & 45.96 & $0.002^{\mathrm{a}}$ \\
\hline & $A C+S C$ & 77.03 & 63.33 & 41.89 & 38.29 & 9.28 & 40.53 & $<0.001^{b}$ \\
\hline
\end{tabular}

Reconstruction parameters: 35 iterations, 20 subsets, no filter; Acquisition parameters: $120 \mathrm{~s} /$ frame of acquisition time, 15\% energy window; $A C$, attenuation correction; $S C$, scatter correction; RR, resolution recovery correction; $a, A C+S C+R R$ vs. $A C+R R ; b, A C+S C+R R$ vs. AC+SC

Table 4 Comparison of COVs (Coefficients of variation, \%) of all spheres among three different correction combinations

\begin{tabular}{|c|c|c|c|c|c|c|c|c|}
\hline & Correction combination & $37 \mathrm{~mm}$ & $28 \mathrm{~mm}$ & $22 \mathrm{~mm}$ & $17 \mathrm{~mm}$ & $13 \mathrm{~mm}$ & $10 \mathrm{~mm}$ & $P$ value \\
\hline \multirow[t]{3}{*}{$32: 1$} & $A C+S C+R R$ & 93.77 & 92.21 & 88.01 & 87.85 & 85.95 & 86.64 & \\
\hline & $A C+R R$ & 74.53 & 72.91 & 70.50 & 71.00 & 69.76 & 70.82 & $<0.001^{\mathrm{a}}$ \\
\hline & $A C+S C$ & 102.96 & 103.52 & 101.71 & 101.53 & 100.70 & 100.80 & $<0.001^{b}$ \\
\hline \multirow[t]{3}{*}{$16: 1$} & $A C+S C+R R$ & 95.09 & 94.61 & 89.02 & 89.62 & 88.86 & 85.71 & \\
\hline & $A C+R R$ & 76.36 & 75.60 & 72.28 & 72.54 & 70.40 & 69.06 & $<0.001^{\mathrm{a}}$ \\
\hline & $A C+S C$ & 96.95 & 96.33 & 97.64 & 97.13 & 95.44 & 94.43 & $0.007^{b}$ \\
\hline \multirow[t]{3}{*}{$8: 1$} & $A C+S C+R R$ & 97.25 & 94.93 & 91.36 & 90.93 & 90.74 & 87.10 & \\
\hline & $A C+R R$ & 77.98 & 75.92 & 73.78 & 72.54 & 72.06 & 69.67 & $<0.001^{\mathrm{a}}$ \\
\hline & $A C+S C$ & 96.31 & 93.75 & 94.79 & 94.89 & 95.34 & 95.60 & $0.096^{\mathrm{b}}$ \\
\hline \multirow[t]{3}{*}{$4: 1$} & $A C+S C+R R$ & 92.71 & 88.97 & 84.03 & 83.13 & 82.02 & 79.55 & \\
\hline & $A C+R R$ & 73.12 & 72.69 & 69.53 & 68.38 & 66.97 & 64.92 & $<0.001^{\mathrm{a}}$ \\
\hline & $A C+S C$ & 91.77 & 91.38 & 91.91 & 91.49 & 91.80 & 94.04 & $0.026^{b}$ \\
\hline
\end{tabular}

Reconstruction parameters: 35 iterations, 20 subsets, no filter; Acquisition parameters: $120 \mathrm{~s} /$ frame of acquisition time, $15 \%$ energy window; $A C$, attenuation correction; $S C$, scatter correction; RR, resolution recovery correction; $a, A C+S C+R R$ vs. $A C+R R ; b, A C+S C+R R v s . A C+S C$

Table 5 Comparison of RCs (\%) between the 15\% energy window and 20\% energy window

\begin{tabular}{lllllllll}
\hline & $\begin{array}{l}\text { Energy } \\
\text { window (\%) }\end{array}$ & $\mathbf{3 7} \mathbf{~} \mathbf{m}$ & $\mathbf{2 8} \mathbf{~} \mathbf{m}$ & $\mathbf{2 2} \mathbf{~} \mathbf{m}$ & $\mathbf{1 7} \mathbf{~} \mathbf{m}$ & $\mathbf{1 3} \mathbf{~ m}$ & $\mathbf{1 0} \mathbf{~ m m}$ & $P$ value \\
\hline $32: 1$ & 15 & 106.70 & 94.94 & 73.44 & 71.06 & 74.39 & 67.80 & 0.023 \\
& 20 & 105.50 & 94.23 & 72.93 & 70.83 & 72.89 & 65.26 & \\
$16: 1$ & 15 & 106.57 & 97.53 & 75.37 & 76.64 & 63.44 & 75.91 & 0.314 \\
& 20 & 104.94 & 96.93 & 75.36 & 77.38 & 63.79 & 74.35 & \\
$8: 1$ & 15 & 103.58 & 96.65 & 73.31 & 70.98 & 73.14 & 54.67 & 0.919 \\
& 20 & 102.46 & 96.73 & 74.10 & 74.64 & 72.02 & 51.80 & \\
$4: 1$ & 15 & 102.89 & 96.64 & 75.93 & 74.79 & 53.82 & 67.59 & 0.933 \\
& 20 & 104.26 & 96.31 & 79.16 & 77.13 & 57.10 & 58.69 & \\
\hline
\end{tabular}

Reconstruction parameters: 35 iterations, 20 subsets, No filter; AC + SC + RR correction. Acquisition parameters: 120 s/frame of acquisition time 

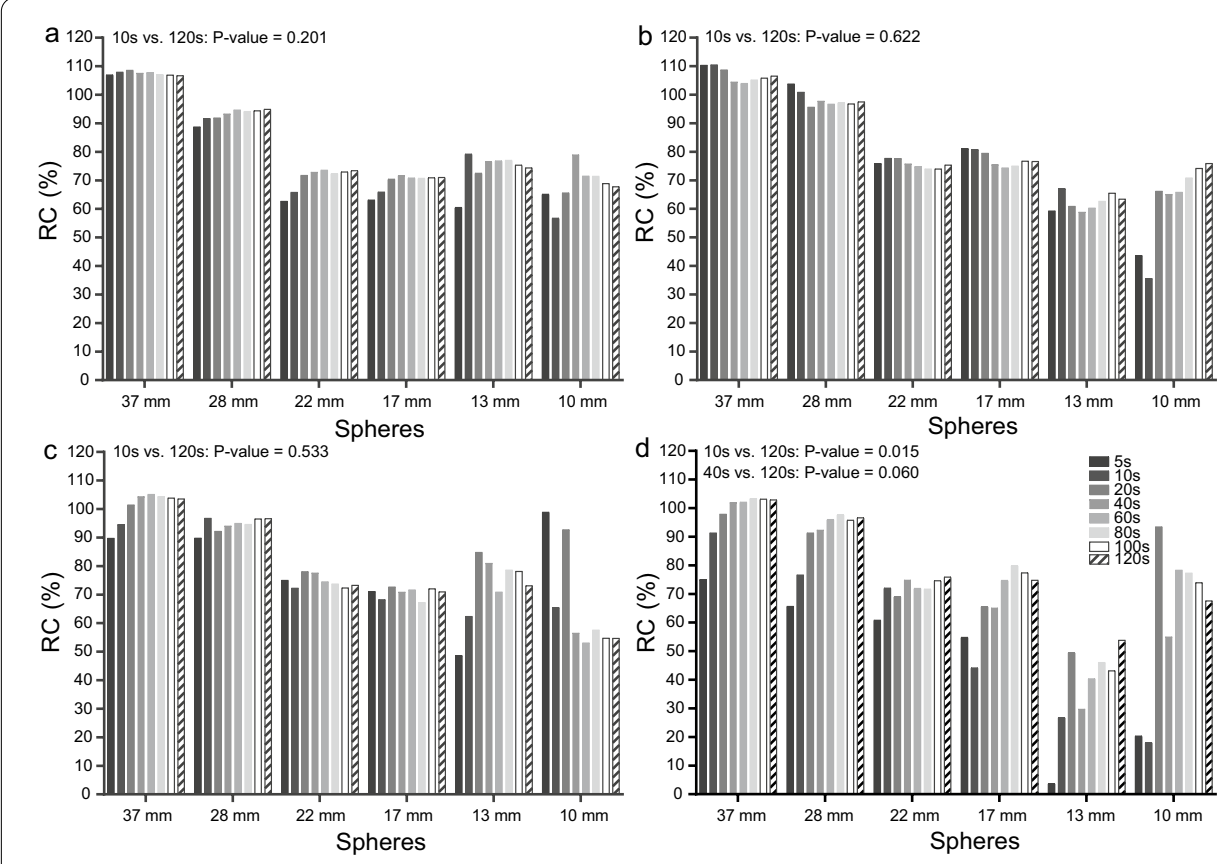

Fig. 7 RCs under different acquisition time. a 32:1 T/B ratio; b 16:1 T/B ratio; c 8:1 T/B ratio; d 4:1 T/B ratio; $X$-axis indicates different spheres grouped by size; $Y$-axis indicates RCs; RCs of each sphere were calculated with 5-120 s/frame of acquisition time (from left to right). $P$ values were calculated by using the paired t-test. Fixed reconstruction parameters: 35 iterations, 20 subsets, no filter, AC+SC+RR combination; Fixed acquisition parameters: $15 \%$ energy window

\section{Discussion}

In this study, CT images were applied as references to avoid the partial volume effect of the SPECT system to calculate RCs with lower errors [58]. The study of Dr. Koole, M et al. suggested that high-resolution structural information from MR or CT images is helpful in determining potential lesions in SPECT images [59].

This study showed that the number of iterations had a large impact on quantification. Figure 2 shows that RCs of larger spheres $(37-17 \mathrm{~mm}$ ) converged earlier than those of smaller spheres (13 $\mathrm{mm}$ and $10 \mathrm{~mm}$ spheres). This indicated that a small number of iterations might be enough for larger lesions in absolute quantification. Although the correlations between RCs and 1-35 iterations were lower than those of 35-90 iterations, 1-35 iterations had much higher regression coefficients than those of 35-90 iterations (Table 1). RCs could also increase rapidly within the first 35 iterations for all spheres (Fig. 2). This indicated that although 35-90 iterations had strong linearity, it could not increase RCs efficiently because of the much smaller regression coefficients compared with those of 1-35 iterations. Furthermore, smaller spheres had relatively larger errors, and more iterations could increase RCs, but not efficiently. Therefore, it was determined that the optimal number of iterations might be 35 .

The correlations between subsets and RCs were not obvious because, for many spheres, the $P$ values were greater than 0.05 (Table 1 ). Additionally, the regression coefficients of all $\mathrm{T} / \mathrm{B}$ ratios were very low $(0.09-0.70$ for the 32:1 T/B ratio, 0.071.14 for the $16: 1 \mathrm{~T} / \mathrm{B}$ ratio, $0.08-0.90$ for the $8: 1 \mathrm{~T} / \mathrm{B}$ ratio, and -0.06 to 1.14 for the 4:1 T/B ratio) (Table 1 ). These results indicated that $\mathrm{RCs}$ could not increase rapidly 

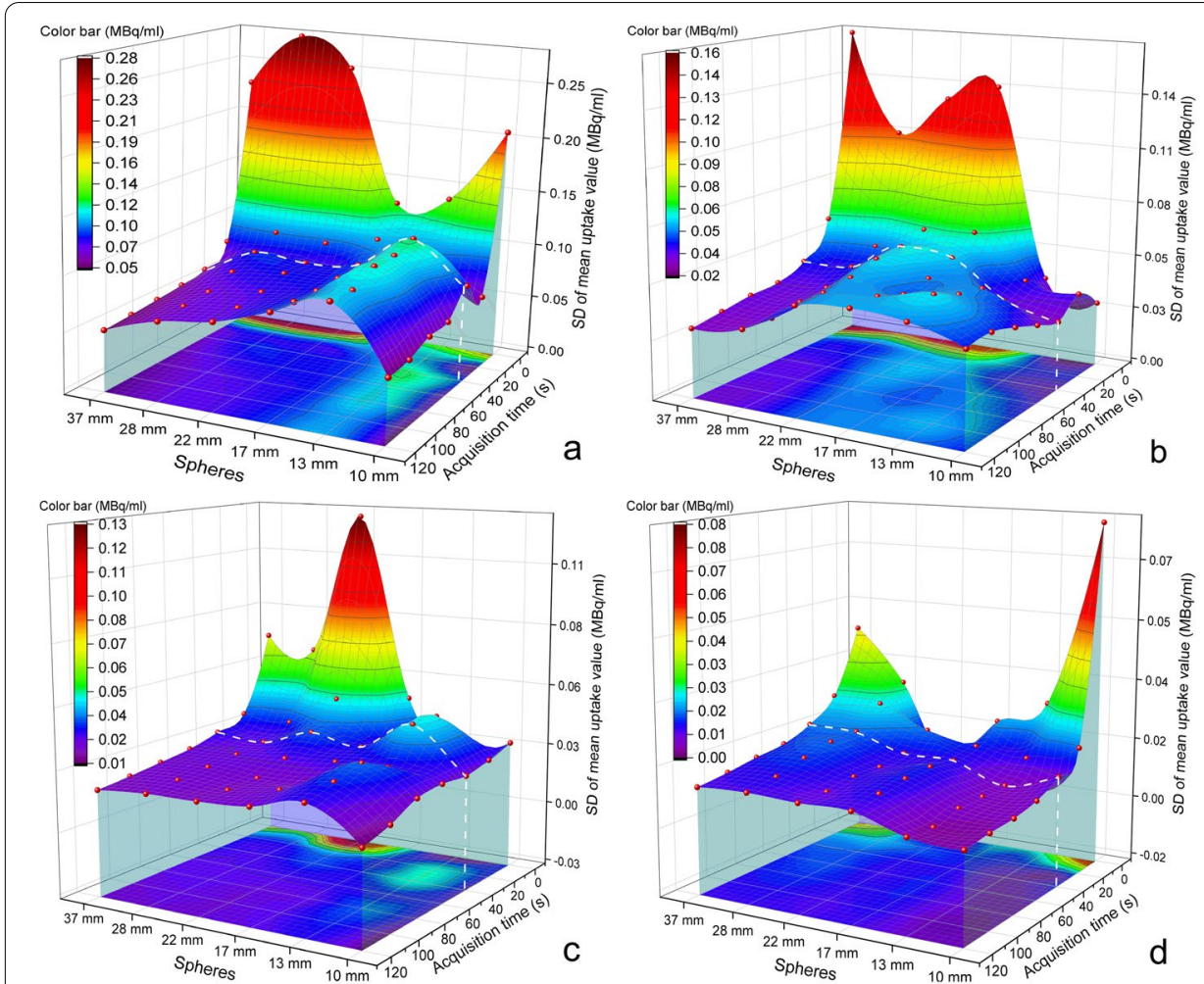

Fig. 8 Fitted 3D-scatter plot of standard deviations (SDs) of $R C$ s. a 32:1 T/B ratio; $\mathbf{b}$ 16:1 T/B ratio; c 8:1 T/B ratio; $\mathbf{d}$ 4:1 T/B ratio; X-axis indicates 6 spheres $(37 \mathrm{~mm}, 28 \mathrm{~mm}, 22 \mathrm{~mm}, 17 \mathrm{~mm}, 13 \mathrm{~mm}$, and $10 \mathrm{~mm}$ ); Y-axis indicates SD of RCs; Z-axis indicates different acquisition time/frame (1-120 s/frame); White dashed line is the proposed cut-off value for acquisition time ( $40 \mathrm{~s} /$ frame). Fixed reconstruction parameters: 35 iterations, 20 subsets, no filter, AC + SC + RR combination; Fixed acquisition parameters: 15\% energy window

with the increasing number of subsets; therefore, subsets had a relatively small impact on quantification. The study of Dr. Vriens, D et al. also suggested that subsets have only a small effect on the standardized uptake value (SUV) in phantom experiments [60]. In this study, RCs tended to be stable after 20 subsets for the larger spheres (37-17 $\mathrm{mm}$ ) and did not increase significantly after 20 subsets for the smaller spheres (13 $\mathrm{mm}$ and $10 \mathrm{~mm}$ spheres). Therefore, 20 subsets were applied in this study.

Among all reconstruction parameters, the FWHM of the Gaussian filter showed the most significant correlations (all Pearson's $r<-0.85$, all $P$ values $<0.05$ ) with RCs as well as the highest regression coefficients $(-9.49$ to -11.83 for the $32: 1 \mathrm{~T} / \mathrm{B}$ ratio, -8.68 to -11.83 for the $16: 1 \mathrm{~T} / \mathrm{B}$ ratio, -6.23 to -10.90 for the $8: 1 \mathrm{~T} / \mathrm{B}$ ratio, -4.23 to -9.39 for the $4: 1 \mathrm{~T} / \mathrm{B}$ ratio, respectively, Table 1$)$. This indicated that the Gaussian filter had a large impact on quantifications. This study showed that there was no plateau of FWHM in terms of RCs, and RCs decreased dramatically along with the decrease of FWHM in all T/B ratios. Since there was no plateau for the Gaussian filter in terms of RCs, it was not used in the following analysis.

The $\mathrm{AC}+\mathrm{SC}+\mathrm{RR}$ combination presented a higher concentration concordance in spheres, and the $\mathrm{AC}+\mathrm{SC}$ combination resulted in the lowest RCs (Fig. 5 and Table 2). Although RCs calculated with the AC+RR combination were higher than those of 
Table 6 RCs (\%) of different acquisition time

\begin{tabular}{|c|c|c|c|c|c|c|c|}
\hline & Time (s/frame) & $37 \mathrm{~mm}$ & $28 \mathrm{~mm}$ & $22 \mathrm{~mm}$ & $17 \mathrm{~mm}$ & $13 \mathrm{~mm}$ & $10 \mathrm{~mm}$ \\
\hline \multirow[t]{8}{*}{$32: 1$} & 5 & 106.95 & 88.73 & 62.68 & 63.13 & 60.54 & 65.16 \\
\hline & 10 & 107.95 & 91.69 & 65.81 & 65.90 & 79.24 & 56.79 \\
\hline & 20 & 108.60 & 91.89 & 71.77 & 70.44 & 72.52 & 65.60 \\
\hline & 40 & 107.65 & 93.33 & 72.92 & 71.78 & 76.66 & 79.02 \\
\hline & 60 & 107.80 & 94.66 & 73.57 & 70.86 & 76.84 & 71.53 \\
\hline & 80 & 107.03 & 94.16 & 72.40 & 70.76 & 77.06 & 71.47 \\
\hline & 100 & 106.92 & 94.40 & 72.97 & 70.94 & 75.35 & 68.91 \\
\hline & 120 & 106.70 & 94.94 & 73.44 & 71.06 & 74.39 & 67.80 \\
\hline \multirow[t]{8}{*}{$16: 1$} & 5 & 110.31 & 103.77 & 75.90 & 81.12 & 59.25 & 43.66 \\
\hline & 10 & 110.49 & 100.87 & 77.71 & 80.73 & 67.05 & 35.59 \\
\hline & 20 & 108.68 & 95.65 & 77.63 & 79.50 & 60.86 & 66.15 \\
\hline & 40 & 104.55 & 97.85 & 75.80 & 75.59 & 58.92 & 65.15 \\
\hline & 60 & 103.98 & 96.69 & 74.82 & 74.33 & 60.26 & 65.82 \\
\hline & 80 & 105.21 & 97.14 & 74.00 & 75.02 & 62.69 & 70.83 \\
\hline & 100 & 105.83 & 96.80 & 73.98 & 76.75 & 65.52 & 74.21 \\
\hline & 120 & 106.57 & 97.53 & 75.37 & 76.64 & 63.44 & 75.91 \\
\hline \multirow[t]{8}{*}{$8: 1$} & 5 & 89.75 & 89.79 & 74.99 & 71.09 & 48.65 & 98.87 \\
\hline & 10 & 94.57 & 96.78 & 72.29 & 68.26 & 62.37 & 65.46 \\
\hline & 20 & 101.43 & 92.18 & 78.06 & 72.69 & 84.84 & 92.74 \\
\hline & 40 & 104.44 & 94.10 & 77.68 & 70.95 & 81.08 & 56.57 \\
\hline & 60 & 105.14 & 94.96 & 74.50 & 71.64 & 70.88 & 53.06 \\
\hline & 80 & 104.39 & 94.64 & 73.74 & 67.25 & 78.64 & 57.59 \\
\hline & 100 & 103.85 & 96.58 & 72.37 & 72.04 & 78.16 & 54.77 \\
\hline & 120 & 103.58 & 96.65 & 73.31 & 70.98 & 73.14 & 54.67 \\
\hline \multirow[t]{8}{*}{$4: 1$} & 5 & 75.02 & 65.68 & 60.85 & 54.83 & 3.72 & 20.36 \\
\hline & 10 & 91.33 & 76.63 & 72.05 & 44.15 & 26.79 & 18.01 \\
\hline & 20 & 97.88 & 91.30 & 69.08 & 65.61 & 49.50 & 93.46 \\
\hline & 40 & 102.02 & 92.37 & 74.92 & 65.15 & 29.80 & 55.07 \\
\hline & 60 & 102.09 & 96.05 & 71.97 & 74.78 & 40.34 & 78.31 \\
\hline & 80 & 103.36 & 97.70 & 71.71 & 79.94 & 46.03 & 77.28 \\
\hline & 100 & 103.17 & 95.81 & 74.64 & 77.40 & 43.13 & 73.95 \\
\hline & 120 & 102.89 & 96.64 & 75.93 & 74.79 & 53.82 & 67.59 \\
\hline
\end{tabular}

Reconstruction parameters: 35 iterations, 20 subsets, No filter; AC + SC + RR correction. Acquisition parameters: 15\% energy window

the $\mathrm{AC}+\mathrm{SC}+\mathrm{RR}$ and $\mathrm{AC}+\mathrm{SC}$ combinations in all spheres, it resulted from the compensation of scattered photons with inaccurate energy and position information. For RCs of the largest $37 \mathrm{~mm}$ sphere calculated with the AC+RR combination in all $\mathrm{T} / \mathrm{B}$ ratios, the plus tolerances were even greater than $20 \%$. In contrast, these plus tolerances were only approximately $2.89-6.70 \%$ for the AC+SC+RR combination (Table 2). Since the scattered photons account for $30-40 \%$ of all photons acquired by the SPECT detector, the application of SC can reduce the errors of the calculated concentration to a great extent [61].

In principle, and among other factors, image quality in nuclear medicine is mainly affected by three factors: (1) spatial resolution (image sharpness) [62], (2) noise (variations in the image due to random effects such as quantum noise) [63], and (3) contrast (difference in image intensity between areas of the imaged object) [64]. The per cent 
contrast only measures the contrast aspect of the images but does not measure noise or spatial resolution, which altogether affects the image quality, as measured by lesion detectability. The resolution in this study was unchanged since the phantom did not have much anatomical variability. Thus, we added a noise evaluation (COV analysis) to the study in addition to the per cent contrast analysis to make the study more comprehensive and the results more indicative or potentially applicable to clinical situations. For image quality, the combination of $\mathrm{AC}+\mathrm{SC}+\mathrm{RR}$ had the best per cent contrasts in all $\mathrm{T} / \mathrm{B}$ ratios (Table 3 , all $P$ values $<0.05$ ). However, the COVs of the $\mathrm{AC}+\mathrm{SC}+\mathrm{RR}$ combination were higher than those of the $\mathrm{AC}+\mathrm{RR}$ combination at all $\mathrm{T} / \mathrm{B}$ ratios (Table 4, all $P$ values $<0.05$ ). The study of Knoll et al. also showed a similar result that the application of SC might increase the background variability [64]. This indicated that although $\mathrm{AC}+\mathrm{SC}+\mathrm{RR}$ combination resulted in the best quantitative performance, its image quality might be somewhat debatable. Since quantification was the main aim of this study, we selected $\mathrm{AC}+\mathrm{SC}+\mathrm{RR}$ combination as the optimal correction combination. Several reports also suggested the significance of AC, SC, and RR for SPECT quantification [29, 65-67]. Our study also showed that for all T/B ratios, COVs were relatively high. This was an inevitable compromise when quantification was the main aim of this study since a larger number of iterations could not only increase quantification accuracy but also increase background noise $[47,68]$.

For the $32: 1 \mathrm{~T} / \mathrm{B}$ ratio, $\mathrm{RCs}$ under the $15 \%$ energy window were higher than those under the $20 \%$ energy window, and there was a statistically significant difference $(P$ value $=0.023)$. However, for lower $T / B$ ratios $(16: 1,8: 1$, and 4:1), there were no statistically significant differences (all $P$ values $>0.05$, Table 5 ). This suggested that although CZT SPECT/CT has a better image resolution due to the improved energy resolution of the new solid-state crystals [69], for RCs, the advantage of the $15 \%$ energy window might not be obvious enough for lower T/B ratios compared with that of the $20 \%$ energy window. Since a $15 \%$ energy window might improve the quantification accuracy for a higher $\mathrm{T} / \mathrm{B}$ ratio (32:1), it was determined to be the optimal energy window in this step.

This study showed that the correlations between RCs and acquisition time were not obvious compared with those of other parameters. The regression coefficients between RCs and the acquisition time/frame were relatively small ( -0.01 to 0.07 for the $32: 1 \mathrm{~T} / \mathrm{B}$ ratio, -0.04 to 0.28 for the $16: 1 \mathrm{~T} / \mathrm{B}$ ratio, -0.31 to 0.12 for the $8: 1 \mathrm{~T} / \mathrm{B}$ ratio, and -0.34 to 0.26 for the $4: 1 \mathrm{~T} / \mathrm{B}$ ratio, respectively, Table 1 ). RCs did not increase significantly with increasing acquisition time/frame (all $P$ values $>0.05$ when comparing $10 \mathrm{~s}$ with $120 \mathrm{~s}$ of acquisition time/frame). However, for the 4:1 T/B ratio, RCs of six spheres increased significantly within the first $40 \mathrm{~s} /$ frame of acquisition time $(10 \mathrm{~s}$ vs. $120 \mathrm{~s}, P$ value $=0.015$; $40 \mathrm{~s}$ vs. $120 \mathrm{~s}, P$ value $=0.060$, Fig. 7). This suggested that for lower concentrations, the optimal acquisition time might be dependent on the activity concentration. Meanwhile, SD could be rapidly reduced within the first $40 \mathrm{~s} /$ frame of acquisition time (Fig. 8). These results suggested that acquisition time might only impose a strong impact on the quantification accuracy within this range. Therefore, $40 \mathrm{~s} /$ frame might be the optimal value in this step. In practice, $40 \mathrm{~s} /$ frame of acquisition time might be able to satisfy the quantification requirement.

The RCs of the $37 \mathrm{~mm}$ sphere reached 102.02-107.65\% under the best acquisition and reconstruction parameters. However, these numbers dropped dramatically as the sphere 
volumes decreased (55.07-79.02\% in the $10 \mathrm{~mm}$ sphere, Table 6). One possible reason is that the VOI counts decreased significantly with smaller objects due to the limitations brought on by the spatial resolution of SPECT [70].

There were four limitations to this study. First, the suggested acquisition and reconstruction parameters might only be applicable for quantitative purposes and for the CZT-SPECT equipment we investigated in this study. Second, due to the purpose of calculating mean uptake values with the lowest errors and determining the impacts of different acquisition and reconstruction parameters, VOIs were delineated using CT images as references. This procedure might be limited in clinical usage. Third, this study also showed that for all T/B ratios, COVs were relatively high. This was an inevitable compromise when quantification was the main aim of this study since a larger number of iterations could not only increase quantification accuracy but also increase background noise. Last, quantification measurements were only performed with a CZT-based camera system and not with a $\mathrm{NaI}(\mathrm{Tl})$-based camera system, so a comparison between them was not evaluated.

\title{
Conclusions
}

CZT-SPECT/CT of technetium showed a good quantification accuracy. The favourable acquisition parameters may be the $15 \%$ energy window and $40 \mathrm{~s} /$ frame. The favourable reconstruction parameters could be 35 iterations, 20 subsets, the $\mathrm{AC}+\mathrm{SC}+\mathrm{RR}$ correction combination, and no filter. Our results might have some merit for clinical quantification guidelines.

\begin{abstract}
Abbreviations
SPECT: Single-photon emission computed tomography; CZT: Cadmium-zinc-telluride; RC: Recovery coefficient; SD: Standard deviation; NEMA: National Electrical Manufacturers Association; FWHM: Full width at half maximum; AC: Attenuation correction; SC: Scatter correction; RR: Resolution recovery correction; PVC: Partial volume correction; Nal: Sodium iodide; PMT: Photomultiplier tube; PET: Positron emission computed tomography; VOl: Volume of interest; ROI: Region of interest; COV: Coefficient of variation; OSEM: Ordered subsets expectation maximization.
\end{abstract}

\section{Supplementary Information}

The online version contains supplementary material available at https://doi.org/10.1186/s40658-021-00412-4.

Additional file 1: Fig. S1 NEMA/IEC 2001 phantom. Black arrows, spheres with different diameters; White arrows, D-shaped cylinder

Acknowledgements

The authors thank Dr. Jiahua Xu, Dr. Yuchao Xu, and Dr. Hong Li, who had always been a source of encouragement and inspiration.

\section{Authors' contributions}

RZ, MW contributed to the design of this study. Material preparation, data collection, and analysis were performed by RZ. The first draft of the manuscript was written by RZ. All authors contributed to manuscript revision, read, approved the submitted version, and agreed to be accountable for all aspects of the research in ensuring the accuracy of this study. All authors read and approved the final manuscript.

\section{Funding}

The design of this study was funded by the National Natural Science Foundation of China grants (Grant Number: \#81571709 and \#81971650). The analysis and interpretation of data of this study were funded by the Key Project of Tianjin Science and Technology Committee Foundation grant (Grant Number: \#16JCZDJC34300).

\section{Availability of data and materials}

The datasets used and/or analysed during the current study are available from the corresponding authors on reasonable request. 


\section{Declarations}

Ethics approval and consent to participate

Not applicable.

\section{Consent for publication}

Not applicable.

\section{Competing interests}

The authors declare that they have no competing interests.

Received: 31 January 2021 Accepted: 10 September 2021

Published online: 26 September 2021

\section{References}

1. Hutton BF. The origins of SPECT and SPECT/CT. Eur J Nucl Med Mol Imaging. 2014;41(Suppl 1):S3-16. https://doi.org/ 10.1007/s00259-013-2606-5

2. Chen J, Garcia EV, Bax JJ, Iskandrian AE, Borges-Neto S, Soman P. SPECT myocardial perfusion imaging for the assessment of left ventricular mechanical dyssynchrony. J Nucl Cardiol: Off Publ Am Soc Nucl Cardiol. 2011;18(4):685-94. https://doi.org/10.1007/s12350-011-9392-x.

3. Wong KK, Fig LM, Youssef E, Ferretti A, Rubello D, Gross MD. Endocrine scintigraphy with hybrid SPECT/CT. Endocr Rev. 2014;35(5):717-46. https://doi.org/10.1210/er.2013-1030.

4. Bajaj N, Hauser RA, Grachev ID. Clinical utility of dopamine transporter single photon emission CT (DaT-SPECT) with (123I) ioflupane in diagnosis of parkinsonian syndromes. J Neurol Neurosurg Psychiatry. 2013;84(11):1288-95. https://doi.org/10.1136/jnnp-2012-304436.

5. Ljungberg M, Pretorius PH. SPECT/CT: an update on technological developments and clinical applications. Br Radiol. 2018;91(1081):20160402. https://doi.org/10.1259/bjr.20160402.

6. Parks ET. Digital radiographic imaging: is the dental practice ready? J Am Dental Assoc (1939). 2008;139(4):477-81. https://doi.org/10.14219/jada.archive.2008.0191.

7. Lee JS, Kovalski G, Sharir T, Lee DS. Advances in imaging instrumentation for nuclear cardiology. J Nucl Cardiol: Off Publ Am Soc Nucl Cardiol. 2019;26(2):543-56. https://doi.org/10.1007/s12350-017-0979-8.

8. Lima R, Peclat T, Soares T, Ferreira C, Souza AC, Camargo G. Comparison of the prognostic value of myocardial perfusion imaging using a CZT-SPECT camera with a conventional anger camera. J Nucl Cardiol: Off Publ Am Soc Nucl Cardiol. 2017;24(1):245-51. https://doi.org/10.1007/s12350-016-0618-9.

9. Slomka PJ, Patton JA, Berman DS, Germano G. Advances in technical aspects of myocardial perfusion SPECT imaging. J Nucl Cardiol: Off Publ Am Soc Nucl Cardiol. 2009;16(2):255-76. https://doi.org/10.1007/s12350-009-9052-6.

10. Ben-Haim S, Kennedy J, Keidar Z. Novel cadmium zinc telluride devices for myocardial perfusion imaging-technological aspects and clinical applications. Semin Nucl Med. 2016;46(4):273-85. https://doi.org/10.1053/j.semnuclmed. 2016.01.002.

11. Lortie M, Beanlands RS, Yoshinaga K, Klein R, Dasilva JN, DeKemp RA. Quantification of myocardial blood flow with 82Rb dynamic PET imaging. Eur J Nucl Med Mol Imaging. 2007;34(11):1765-74. https://doi.org/10.1007/ s00259-007-0478-2

12. Bai B, Bading J, Conti PS. Tumor quantification in clinical positron emission tomography. Theranostics. 2013;3(10):787-801. https://doi.org/10.7150/thno.5629.

13. Ritt P, Vija H, Hornegger J, Kuwert T. Absolute quantification in SPECT. Eur J Nucl Med Mol Imaging. 2011;38(Suppl 1):S69-77. https://doi.org/10.1007/s00259-011-1770-8.

14. Lindemann ME, Guberina N, Wetter A, Fendler WP, Jakoby B, Quick HH. Improving (68)Ga-PSMA PET/MRI of the prostate with unrenormalized absolute scatter correction. J Nucl Med: Off Publ Soc Nucl Med. 2019;60(11):1642-8. https://doi.org/10.2967/jnumed.118.224139.

15. Koral KF, Wang XQ, Rogers WL, Clinthorne NH, Wang XH. SPECT Compton-scattering correction by analysis of energy spectra. J Nucl Med: Off Publ Soc Nucl Med. 1988;29(2):195-202.

16. Núñez M, Prakash V, Vila R, Mut F, Alonso O, Hutton BF. Attenuation correction for lung SPECT: evidence of need and validation of an attenuation map derived from the emission data. Eur J Nucl Med Mol Imaging. 2009;36(7):1076-89. https://doi.org/10.1007/s00259-009-1090-4.

17. Soret M, Bacharach SL, Buvat I. Partial-volume effect in PET tumor imaging. J Nucl Med: Off Publ Soc Nucl Med. 2007;48(6):932-45. https://doi.org/10.2967/jnumed.106.035774.

18. Pretorius PH, King MA. Diminishing the impact of the partial volume effect in cardiac SPECT perfusion imaging. Med Phys. 2009;36(1):105-15. https://doi.org/10.1118/1.3031110.

19. Schelbert HR, Hoh CK, Royal HD, Brown M, Dahlbom MN, Dehdashti F, et al. Procedure guideline for tumor imaging using fluorine-18-FDG. J Nucl Med: Off Publ Soc Nucl Med. 1998;39(7):1302-5.

20. Boellaard R. Standards for PET image acquisition and quantitative data analysis. J Nucl Med: Off Publ Soc Nucl Med. 2009;50(Suppl 1):11s-20s. https://doi.org/10.2967/jnumed.108.057182.

21. Axelsson B, Msaki P, Israelsson A. Subtraction of Compton-scattered photons in single-photon emission computerized tomography. J Nucl Med: Off Publ Soc Nucl Med. 1984;25(4):490-4.

22. Floyd CE Jr, Jaszczak RJ, Greer KL, Coleman RE. Deconvolution of Compton scatter in SPECT. J Nucl Med: Off Publ Soc Nucl Med. 1985;26(4):403-8.

23. Jaszczak RJ, Greer KL, Floyd CE Jr, Harris CC, Coleman RE. Improved SPECT quantification using compensation for scattered photons. J Nucl Med: Off Publ Soc Nucl Med. 1984;25(8):893-900. 
24. Lowry CA, Cooper MJ. The problem of Compton scattering in emission tomography: a measurement of its spatial distribution. Phys Med Biol. 1987;32(9):1 187-91. https://doi.org/10.1088/0031-9155/32/9/013.

25. DeVito RP, Hamill JJ. Determination of weighting functions for energy-weighted acquisition. J Nucl Med: Off Publ Soc Nucl Med. 1991;32(2):343-9.

26. Halama JR, Henkin RE, Friend LE. Gamma camera radionuclide images: improved contrast with energy-weighted acquisition. Radiology. 1988;169(2):533-8. https://doi.org/10.1148/radiology.169.2.3262885.

27. Floyd CE Jr, Jaszczak RJ, Greer KL, Coleman RE. Inverse Monte Carlo as a unified reconstruction algorithm for ECT. J Nucl Med: Off Publ Soc Nucl Med. 1986;27(10):1577-85.

28. Floyd CE Jr, Jaszczak RJ, Greer KL, Coleman RE. Brain phantom: high-resolution imaging with SPECT and I-123. Radiology. 1987;164(1):279-81. https://doi.org/10.1148/radiology.164.1.3495817.

29. Zeintl J, Vija AH, Yahil A, Hornegger J, Kuwert T. Quantitative accuracy of clinical 99mTc SPECT/CT using orderedsubset expectation maximization with 3-dimensional resolution recovery, attenuation, and scatter correction. J Nucl Med: Off Publ Soc Nucl Med. 2010;51(6):921-8. https://doi.org/10.2967/jnumed.109.071571.

30. Rajeevan N, Zubal IG, Ramsby SQ, Zoghbi SS, Seibyl J, Innis RB. Significance of nonuniform attenuation correction in quantitative brain SPECT imaging. J Nucl Med: Off Publ Soc Nucl Med. 1998;39(10):1719-26.

31. Tsui BM, Frey EC, Zhao X, Lalush DS, Johnston RE, McCartney WH. The importance and implementation of accurate 3D compensation methods for quantitative SPECT. Phys Med Biol. 1994;39(3):509-30. https://doi.org/10.1088/00319155/39/3/015.

32. Liow JS, Strother SC, Rehm K, Rottenberg DA. Improved resolution for PET volume imaging through three-dimensional iterative reconstruction. J Nucl Med: Off Publ Soc Nucl Med. 1997;38(10):1623-31.

33. Müller-Gärtner HW, Links JM, Prince JL, Bryan RN, McVeigh E, Leal JP, et al. Measurement of radiotracer concentration in brain gray matter using positron emission tomography: MRI-based correction for partial volume effects. J Cerebral Blood Flow Metab: Off J Int Soc Cerebral Blood Flow Metab. 1992;12(4):571-83. https://doi.org/10.1038/ jcbfm.1992.81.

34. Huesman RH. A new fast algorithm for the evaluation of regions of interest and statistical uncertainty in computed tomography. Phys Med Biol. 1984;29(5):543-52. https://doi.org/10.1088/0031-9155/29/5/007.

35. Bailey DL, Willowson KP. An evidence-based review of quantitative SPECT imaging and potential clinical applications. J Nucl Med: Off Publ Soc Nucl Med. 2013;54(1):83-9. https://doi.org/10.2967/jnumed.112.111476.

36. Kaneta T. PET and SPECT imaging of the brain: a review on the current status of nuclear medicine in Japan. Jpn J Radiol. 2020;38(4):343-57. https://doi.org/10.1007/s11604-019-00901-8.

37. Peters SMB, van der Werf NR, Segbers M, van Velden FHP, Wierts R, Blokland $K$, et al. Towards standardization of absolute SPECT/CT quantification: a multi-center and multi-vendor phantom study. EJNMMI physics. 2019;6(1):29. https://doi.org/10.1186/s40658-019-0268-5.

38. Seret A, Nguyen D, Bernard C. Quantitative capabilities of four state-of-the-art SPECT-CT cameras. EJNMMI Res. 2012;2(1):45. https://doi.org/10.1186/2191-219x-2-45.

39. Nakahara T, Daisaki H, Yamamoto Y, Iimori T, Miyagawa K, Okamoto T, et al. Use of a digital phantom developed by QIBA for harmonizing SUVs obtained from the state-of-the-art SPECT/CT systems: a multicenter study. EJNMMI Res. 2017;7(1):53. https://doi.org/10.1186/s13550-017-0300-5.

40. He B, Frey EC. Comparison of conventional, model-based quantitative planar, and quantitative SPECT image processing methods for organ activity estimation using In-111 agents. Phys Med Biol. 2006;51(16):3967-81. https://doi. org/10.1088/0031-9155/51/16/006.

41. Siman W, Mikell JK, Kappadath SC. Practical reconstruction protocol for quantitative (90)Y bremsstrahlung SPECT/CT. Med Phys. 2016;43(9):5093. https://doi.org/10.1118/1.4960629.

42. Beauregard JM, Hofman MS, Pereira JM, Eu P, Hicks RJ. Quantitative (177)Lu SPECT (QSPECT) imaging using a commercially available SPECT/CT system. Cancer Imaging: Off Publ Int Cancer Imaging Soc. 2011;11(1):56-66. https:// doi.org/10.1102/1470-7330.2011.0012.

43. Bastiaannet $\mathrm{R}$, van der Velden $\mathrm{S}$, Lam M, Viergever MA, de Jong H. Fast and accurate quantitative determination of the lung shunt fraction in hepatic radioembolization. Phys Med Biol. 2019;64(23): 235002. https://doi.org/10.1088/ 1361-6560/ab4e49.

44. Acampa W, He W, di Nuzzo C, Cuocolo A. Quantification of SPECT myocardial perfusion imaging. J Nucl Cardiol: Off Publ Am Soc Nucl Cardiol. 2002;9(3):338-42. https://doi.org/10.1067/mnc.2002.123917.

45. Collarino A, Pereira Arias-Bouda LM, Valdés Olmos RA, van der Tol P, Dibbets-Schneider P, de Geus-Oei LF, et al. Experimental validation of absolute SPECT/CT quantification for response monitoring in breast cancer. Med Phys. 2018;45(5):2143-53. https://doi.org/10.1002/mp.12880.

46. Veres DS, Máthé D, Futó I, Horváth I, Balázs A, Karlinger K, et al. Quantitative liver lesion volume determination by nanoparticle-based SPECT. Mol Imag Biol. 2014;16(2):167-72. https://doi.org/10.1007/s11307-013-0679-y.

47. Dickson JC, Tossici-Bolt L, Sera T, Erlandsson K, Varrone A, Tatsch K, et al. The impact of reconstruction method on the quantification of DaTSCAN images. Eur J Nucl Med Mol Imaging. 2010;37(1):23-35. https://doi.org/10.1007/ s00259-009-1212-z.

48. Gnesin S, Leite Ferreira P, Malterre J, Laub P, Prior JO, Verdun FR. Phantom validation of Tc-99m absolute quantification in a SPECT/CT commercial device. Comput Math Methods Med. 2016;2016:4360371. https://doi.org/10.1155/ 2016/4360371.

49. Galt JR, Cullom SJ, Garcia EV. SPECT quantification: a simplified method of attenuation and scatter correction for cardiac imaging. J Nucl Med: Off Publ Soc Nucl Med. 1992;33(12):2232-7.

50. Kim KM, Varrone A, Watabe H, Shidahara M, Fujita M, Innis RB, et al. Contribution of scatter and attenuation compensation to SPECT images of nonuniformly distributed brain activities. J Nucl Med: Off Publ Soc Nucl Med. 2003;44(4):512-9.

51. Kato TS, Lippel M, Naka Y, Mancini DM, Schulze PC. Post-transplant survival estimation using pre-operative albumin levels. J Heart Lung Transplant. 2014;33(5):547-8. https://doi.org/10.1016/j.healun.2014.01.921.

52. Hudson HM, Larkin RS. Accelerated image reconstruction using ordered subsets of projection data. IEEE Trans Med Imaging. 1994;13(4):601-9. https://doi.org/10.1109/42.363108. 
53. van Sluis J, de Jong J, Schaar J, Noordzij W, van Snick P, Dierckx R, et al. Performance characteristics of the digital biograph vision PET/CT system. J Nucl Med: Off Publ Soc Nucl Med. 2019;60(7):1031-6. https://doi.org/10.2967/ jnumed.118.215418.

54. Ryu H, Meikle SR, Willowson KP, Eslick EM, Bailey DL. Performance evaluation of quantitative SPECT/CT using NEMA NU 2 PET methodology. Phys Med Biol. 2019;64(14): 145017. https://doi.org/10.1088/1361-6560/ab2a22.

55. Zeraatkar N, Kalluri KS, Auer B, Konik A, Fromme TJ, Furenlid LR, et al. Investigation of axial and angular sampling in multi-detector pinhole-SPECT brain imaging. IEEE Trans Med Imaging. 2020;39(12):4209-24. https://doi.org/10. 1109/tmi.2020.3015079.

56. Li Y, O'Reilly S, Plyku D, Treves ST, Du Y, Fahey F, et al. A projection image database to investigate factors affecting image quality in weight-based dosing: application to pediatric renal SPECT. Phys Med Biol. 2018;63(14): 145004. https://doi.org/10.1088/1361-6560/aacbfo.

57. Chen MK. Paired t test, negative intraclass correlations, and case-control studies. Am J Clin Nutr. 1981;34(5):959-61. https://doi.org/10.1093/ajcn/34.5.959.

58. Chan C, Liu H, Grobshtein Y, Stacy MR, Sinusas AJ, Liu C. Noise suppressed partial volume correction for cardiac SPECT/CT. Med Phys. 2016;43(9):5225. https://doi.org/10.1118/1.4961391.

59. Koole M, Laere KV, de Walle RV, Vandenberghe S, Bouwens L, Lemahieu I, et al. MRI guided segmentation and quantification of SPECT images of the basal ganglia: a phantom study. Comput Med Imaging Graph: Off J Comput Med Imaging Soc. 2001;25(2):165-72. https://doi.org/10.1016/s0895-6111(00)00045-8.

60. Vriens D, Visser EP, de Geus-Oei LF, Oyen WJ. Methodological considerations in quantification of oncological FDG PET studies. Eur J Nucl Med Mol Imaging. 2010;37(7):1408-25. https://doi.org/10.1007/s00259-009-1306-7.

61. Hutton BF, Buvat I, Beekman FJ. Review and current status of SPECT scatter correction. Phys Med Biol. 2011;56(14):R85-112. https://doi.org/10.1088/0031-9155/56/14/r01.

62. Fahey FH, Harkness BA, Keyes JW Jr, Madsen MT, Battisti C, Zito V. Sensitivity, resolution and image quality with a multi-head SPECT camera. J Nucl Med: Off Publ Soc Nucl Med. 1992;33(10):1859-63.

63. He X, Links JM, Frey EC. An investigation of the trade-off between the count level and image quality in myocardial perfusion SPECT using simulated images: the effects of statistical noise and object variability on defect detectability. Phys Med Biol. 2010;55(17):4949-61. https://doi.org/10.1088/0031-9155/55/17/005.

64. Knoll P, Kotalova D, Köchle G, Kuzelka I, Minear G, Mirzaei S, et al. Comparison of advanced iterative reconstruction methods for SPECT/CT. Z Med Phys. 2012;22(1):58-69. https://doi.org/10.1016/j.zemedi.2011.04.007.

65. Cade SC, Arridge S, Evans MJ, Hutton BF. Use of measured scatter data for the attenuation correction of single photon emission tomography without transmission scanning. Med Phys. 2013;40(8): 082506. https://doi.org/10.1118/1. 4812686.

66. Velidaki A, Perisinakis K, Koukouraki S, Koutsikos J, Vardas P, Karkavitsas N. Clinical usefulness of attenuation and scatter correction in TI-201 SPECT studies using coronary angiography as a reference. Hellenic J Cardiol: HJC = Hellenike Kardiologike Epitheorese. 2007:48(4):211-7.

67. Armstrong IS, Saint KJ, Tonge CM, Arumugam P. Evaluation of general-purpose collimators against high-resolution collimators with resolution recovery with a view to reducing radiation dose in myocardial perfusion SPECT: a preliminary phantom study. J Nucl Cardiol: Off Publ Am Soc Nucl Cardiol. 2017;24(2):596-604. https://doi.org/10.1007/ s12350-015-0368-0.

68. Işı $\mathrm{kCI} \mathrm{Nl}$, Abuqbeitah M. Quantitative improvement of lymph nodes visualization of breast cancer using $(99 \mathrm{~m})$ TC-nanocolloid SPECT/CT and updated reconstruction algorithm. Radiat Environ Biophys. 2021. https://doi.org/10. 1007/s00411-021-00914-w.

69. Slomka PJ, Miller RJH, Hu LH, Germano G, Berman DS. Solid-state detector SPECT myocardial perfusion imaging. J Nucl Med: Off Publ Soc Nucl Med. 2019;60(9):1194-204. https://doi.org/10.2967/jnumed.118.220657.

70. Dewaraja YK, Ljungberg M, Koral KF. Accuracy of 1311 tumor quantification in radioimmunotherapy using SPECT imaging with an ultra-high-energy collimator: Monte Carlo study. J Nucl Med. 2000;41(10):1760-7.

\section{Publisher's Note}

Springer Nature remains neutral with regard to jurisdictional claims in published maps and institutional affiliations.

\section{Submit your manuscript to a SpringerOpen ${ }^{\circ}$ journal and benefit from:}

- Convenient online submission

- Rigorous peer review

- Open access: articles freely available online

- High visibility within the field

Retaining the copyright to your article

Submit your next manuscript at $\boldsymbol{\nabla}$ springeropen.com 